University of Zurich

Department of Economics

Working Paper Series

ISSN 1664-7041 (print)

ISSN 1664-705X (online)

Working Paper No. 361

\title{
The n-Player Hirshleifer Contest
}

Christian Ewerhart and Guang-Zhen Sun

Revised version, October 2022 


\title{
The $n$-Player Hirshleifer Contest*
}

\author{
Christian Ewerhart ${ }^{\dagger}$ \\ Guang-Zhen Sun $\ddagger$
}

October 31, 2022

\begin{abstract}
While the game-theoretic analysis of conflict is often based upon the assumption of multiplicative noise, additive noise such as assumed by Hirshleifer (1989) may be equally plausible depending on the application. In this paper, we examine the equilibrium set of the $n$-player difference-form contest with heterogeneous valuations. For high or intermediate levels of noise, the equilibrium is in pure strategies, with at most one player being active. For small levels of noise, however, we find a variety of equilibria in which some but not necessarily all players randomize. In the case of homogeneous valuations, we obtain a partial uniqueness result for symmetric equilibria. As the contest becomes increasingly decisive, at least two contestants bid up to the valuation of the second-ranked contestant, while any others ultimately drop out. In the limit, equilibria in the Hirshleifer contest converge to equilibria in the corresponding all-pay auction.
\end{abstract}

Keywords. Difference-form contest · Nash equilibrium · Rent dissipation · All-pay auction

JEL codes. C72, D72, D74

*) For useful discussions, we are grateful to Dan Kovenock and David Levine.

†) (corresponding) Department of Economics, University of Zurich, Schönberggasse 1, 8001 Zurich, Switzerland; christian.ewerhart@econ.uzh.ch.

†) Department of Economics, University of Macau; gzsun@um.edu.mo. 


\section{Introduction}

Recent years have witnessed a tremendous surge in interest in the game-theoretic analysis of conflict. ${ }^{1}$ Much of this interest has focused on specific classes of contest technologies that admit both a plausible axiomatic characterization and a stochastic foundation. Among those, the technologies introduced by Tullock (1980) and Hirshleifer (1989) figure most prominently as the canonical models for the ratio-form and the difference-form contests, respectively. ${ }^{2}$

The Tullock contest is analytically convenient because its contest technology is homogeneous of degree zero, i.e., what matters for the probability of winning is the ratio of efforts. Moreover, at least two agents are active in any equilibrium, which is certainly appealing in the analysis of conflict. An undesirable implication of that assumption, however, is that the chance of winning by one contestant who bids zero with someone else bidding only slightly above zero vanishes. Difference-form contest, on the other hand, are often criticized on the grounds that their technology is, by definition, unresponsive if both efforts are raised by the same amount, even if that brings the two effort levels on a similar level in relative terms. Notwithstanding, Hirshleifer's technology is arguably more suitable than the ratio form, e.g., for modeling military conflict. $^{3}$ This is due to two remarkable features of the Hirshleifer technology, viz. a positive probability of winning despite exerting a zero effort and increasing returns to marginal effort up to the inflection point where the probability of winning the prize is one half. Indeed, these constituted the major motivation for Hirshleifer (1989, pp. 103-104) to develop an alternative to the ratio-form model.

This paper examines the equilibrium set of the $n$-player Hirshleifer contest with heterogeneous valuations. To discuss the findings, it proves useful to organize the analysis along the decisiveness of the contest, i.e., along the level of noise in the contest technology. For high and

\footnotetext{
${ }^{1}$ For an introduction to the theory of contests, see Konrad (2009). A more recent survey is Corchón and Serena (2018).

${ }^{2}$ See Skaperdas (1996) and Ewerhart (2015a), respectively, for axiom systems applying to a population of varying and constant size. Stochastic foundations of the Tullock contest in terms of multiplicative noise are due to Hirshleifer and Riley (1992) and Jia (2008). See also Jia and Skaperdas (2012) and Jia et al. (2013). An analogous foundation for the Hirshleifer contest in terms of additive noise is known as the multinomial discrete choice model (with i.i.d. Gumbel distributed error terms). See, e.g., Anderson et al. (1992).

${ }^{3}$ For further discussion of the relative merits of Tullock vs. Hirshleifer, see Hirshleifer $(1989,1995,2000)$, Mueller (2003, p. 342), and Garfinkel and Skaperdas (2007, pp. 655-656).
} 
intermediate levels of noise, we find pure-strategy Nash equilibria (PSNE) of two types. In one type of equilibrium, all players exert zero effort. We refer to this equilibrium as "multilateral peace." Indeed, when the contest technology is not very sharp, incentives to exert effort are weak, which makes inactivity an optimal choice. In another type of pure-strategy equilibrium, one player chooses a positive level of effort, while all other contestants remain inactive. This type of equilibrium will be referred to as "one-sided dominance." The intuition here is as follows. As the technology becomes more effective, contestants with relatively high valuations (or, equivalently, relatively low marginal cost of effort) have an incentive to become active. However, once a single contestant grasps this opportunity, the incentives for all the other contestants are weakened, so that they optimally choose to remain inactive. Notably, however, the identity of the active player is not determined by the ranking of the valuations alone, which implies the possibility of multiple, payoff-inequivalent equilibria. These results, which amount to a fairly complete characterization of the set of equilibria in pure strategies, confirm and extend the intuitions derived in prior analyses of the case of two contestants in the literature.

Once the noise in the contest technology reaches a low level, overbidding by a small margin ensures a win with high probability. For instance, contestants with relatively higher valuations may cease to remain inactive by exerting positive efforts, overbidding any dominating bid. However, in contrast to the case of multiplicative noise, at most one player may be active with probability one. Therefore, there are no equilibria in pure strategies anymore. Instead, one player randomizes, or several players randomize. That is, we obtain mixed-strategy Nash equilibria (MSNE). Given the smoothness properties of Hirshleifer's contest success function, contestants randomize over a finite set of strategies. However, the bids and the probabilities with which they are used remain determined by a system of equations that does not admit an explicit solution in general. In the case of homogeneous valuations, and for symmetric equilibria, the number of bids over which contestants randomize tends to increase, and the equilibrium payoff tends to decline, as the noise becomes smaller, which is intuitive. We also obtain a partial uniqueness result in this case, by combining ideas from the literature on zero-sum games with concepts from the theory of montone comparative statics. Allowing for asymmetric equilibria, the equilibrium set becomes convoluted even for the case of homogeneous valuations. 
For example, over a non-degenerate set of the parameter space, some players may choose to remain inactive, while others may choose to randomize. We present a variety of examples that illustrate the large variety of equilibria that this model admits. Further, in the case of heterogeneous valuations, we derive a general inequality that relates the cardinalities of the contestants' equilibrium supports and use it to obtain some structural results in the case where all but one contestant uses a pure strategy.

We go on and study the case of vanishing small noise. In this case, the contest technology approaches that of the standard all-pay auction. It is shown that, as the technology becomes increasingly deterministic, at least two contestants become heavily engaged in the sense that they bid, with positive probability, nearly up to the valuation of the second-ranked contestant. Therefore, the undissipated rent goes to zero for all but the single contestant of the highest valuation (if there is any). Contestants that are not heavily engaged ultimately become inactive in the limit. Finally, we derive a collection of necessary properties of the equilibrium for the case of arbitrarily small noise and show that convergent sequences of MSNE that correspond to an unbounded sequence of the decisiveness parameter ultimately approach a MSNE of the standard all-pay auction.

The analysis is complemented by a brief analysis of the case of large populations. Specifically, keeping the decisiveness parameter fixed, we find that, if the number of contestants $n$ grows sufficiently large, then the unique equilibrium in the $n$-player Hirshleifer contest is multilateral peace. ${ }^{4}$

General classes of difference-form contests, which include the Hirshleifer contest as a special case, have been analyzed for somewhat more than two decades (Baik, 1998). Assuming a uniform distribution of noise, Che and Gale (2000) were the first in comprehensively characterizing MSNE for a class of two-player contests of the difference form. More recently, Cubel and Sanchez-Pages (2020) have generalized that analysis by allowing for more than two contestants and more flexible difference-form contests. ${ }^{5}$ However, none of those papers touches upon the

\footnotetext{
${ }^{4}$ This observation contrasts with the case of the standard $n$-player Tullock contest where, regardless of $n$, all contestants are active in the symmetric PSNE (Corcoran, 1988).

${ }^{5}$ See also Cubel and Sanchez-Pages (2016), who axiomatized difference-form contest success functions more generally.
} 
questions addressed in the present study. In his seminal contribution, Hirshleifer (1989) identified two main types of PSNE between two contestants, viz. bilateral peace and one-sided dominance. He also offered an informal discussion of MSNE for the case of two contestants. Finally, when introducing the $n$-player generalization, he noted the equivalence of representations (1) and (2) below. However, he did not say anything about equilibria in the $n$-player case. In earlier work (Ewerhart and Sun, 2018), we have shown that the two-player Hirshleifer contest with homogeneous valuations generally admits a unique Nash equilibrium. We also provided an explicit characterization of the Nash equilibrium, which is necessarily symmetric. The case of heterogeneous valuations, still with two players, has been analyzed by Ewerhart (2021). However, as far as we know, the equilibrium set of the Hirshleifer contest with more than two contestants has not been studied so far.

A technically important dimension in which the Hirshleifer contest differs from the Tullock contest is that equilibria in the case of two contestants and homogeneous valuations cannot be used as a basis for constructing equilibria in the case of $n$ contestants and heterogeneous valuations. As noted by Alcalde and Dahm (2010), having one of two active contestants with multiplicative noise use the zero bid with positive probability is equivalent to lowering the valuation of the other active player. Using this trick, so-called all-pay auction equilibria may be constructed in generalized Tullock models with heterogeneous valuations. This trick, however, does not work in models with additive noise. The reason is that the marginal payoff against a zero bid is not zero, as it is the case in the interior for the Tullock contest.

The remainder of the paper is structured as follows. Section 2 contains preliminaries. The respective cases of high and intermediate levels of noise and low levels of noise are considered in Sections 3 and 4. Section 5 deals with the limit case of vanishing noise. Section 6 concerns the case of large populations. Section 7 concludes. All technical proofs have been relegated to an Appendix.

\section{Preliminaries}

\subsection{Set-up and notation}

There are $n \geq 2$ contestants (or players), collected in a set $N=\{1, \ldots, n\}$, that exert effort to 
win a single indivisible prize. Contestant $i$ 's valuation of the prize is denoted by $V_{i}>0$. Thus, we allow for heterogeneous valuations of the prize. Without loss of generality, valuations will be ordered in size, i.e., we assume throughout that $V_{1} \geq \ldots \geq V_{n}>0$. Contestant $i$ 's expected payoff in the $n$-player Hirshleifer contest is given as

$$
\Pi_{i}\left(x_{1}, \ldots, x_{n}\right)=\frac{V_{i}}{\sum_{j=1}^{n} \exp \left(\alpha\left(x_{j}-x_{i}\right)\right)}-x_{i}
$$

where $x_{j} \geq 0$, for $j \in N$, denotes contestant $j$ 's effort (or bid), and the parameter $\alpha>0$ measures the decisiveness of the contest technology. It is easy to see that, as $\alpha \rightarrow 0$, the contest converges to the limit case of a pure lottery, where decisions about expenses do not matter and the winner is determined by chance alone. As $\alpha \rightarrow \infty$, however, the vector of payoffs approximates that of the standard all-pay auction, where the highest bidder wins with certainty (Baye et al., 1990, 1996). Thus, intuitively, chance plays a larger role in the determination of the winner when $\alpha$ is small.

Rewriting relationship (1), one obtains the equivalent logit representation of contestant $i$ 's expected payoff as

$$
\Pi_{i}\left(x_{1}, \ldots, x_{n}\right)=\frac{\exp \left(\alpha x_{i}\right) V_{i}}{\sum_{j=1}^{n} \exp \left(\alpha x_{j}\right)}-x_{i}
$$

It is noteworthy that the impact function $x_{i} \mapsto X_{i} \equiv \exp \left(\alpha x_{i}\right)$ exhibits strictly increasing returns, i.e., it is strictly convex, for all values of $\alpha .^{6}$

As usual, a pure-strategy Nash equilibrium (PSNE) is a vector of bids, $x^{*}=\left(x_{1}^{*}, \ldots, x_{n}^{*}\right) \in$ $\mathbb{R}_{\geq 0}^{n}$, such that $\Pi_{i}^{*} \equiv \Pi_{i}\left(x_{i}^{*}, x_{-i}^{*}\right) \geq \Pi_{i}\left(x_{i}, x_{-i}^{*}\right)$ holds for any $i \in N$ and $x_{i} \in \mathbb{R}_{\geq 0}$, where we adhere to the convention that $x^{*}=\left(x_{i}^{*}, x_{-i}^{*}\right)$, etc.

We will also allow for equilibria in randomized strategies. By a mixed strategy for contestant $i$, we mean a probability measure $\mu_{i}$ on (the Borel subsets of) the interval $\left[0, V_{i}\right]$. Note that the upper bound is introduced without loss of generality because any effort level exceeding a player's valuation is strictly dominated by the zero bid. Let $M_{i}$ denote the set of mixed strategies for contestant $i$. Pure strategies $x_{i} \in\left[0, V_{i}\right]$ are understood as Dirac probability measures, as usual.

\footnotetext{
${ }^{6}$ In contests between more than two parties, increasing returns suggest a motive to form alliances. However, as discussed by Garfinkel and Skaperdas (2007, Sec. 7), the prospect of free-riding and intragroup conflict might render such alliances instable. Further, if alliances are stable, then parties might be able to avoid the contest altogether (by forming a grand coalition).
} 
Contestant $i$ 's expected payoff from a mixed-strategy profile $\mu=\left(\mu_{i}, \mu_{-i}\right) \in M \equiv M_{1} \times \ldots \times M_{n}$ will be written as $E_{\left(\mu_{i}, \mu_{-i}\right)}\left[\Pi_{i}\left(x_{i}, x_{-i}\right)\right]$. A mixed-strategy Nash equilibrium (MSNE) is then a tuple $\mu^{*}=\left(\mu_{1}^{*}, \ldots, \mu_{n}^{*}\right) \in M$ such that $E_{\left(\mu_{i}^{*}, \mu_{-i}^{*}\right)}\left[\Pi_{i}\left(x_{i}, x_{-i}\right)\right] \geq E_{\left(\mu_{i}, \mu_{-i}^{*}\right)}\left[\Pi_{i}\left(x_{i}, x_{-i}\right)\right]$ holds true for any $i \in N$ and $\mu_{i} \in M_{i}$. Existence of an equilibrium in randomized strategies is always guaranteed.

Lemma 1. (Existence) For any $n \geq 2$, the $n$-player Hirshleifer contest with parameter $\alpha>0$ and valuations $V_{1} \geq \ldots \geq V_{n}>0$ admits a MSNE $\mu^{*}$.

Proof. See the Appendix.

\subsection{An initial observation}

We prepare the main analysis by making a general observation. We say that contestant $i \in N$ is active (always active, inactive) in a mixed-strategy profile $\mu \in M$ if her strategy $\mu_{i}$ employs positive bids with positive probability (with probability one, with probability zero). ${ }^{7}$

Lemma 2. (Activity) At most one contestant is always active.

Proof. See the Appendix.

Thus, in any equilibrium, either all contestants choose the zero bid with positive probability, or there is precisely one contestant that is always active. We will see below that both cases are in fact feasible. Thus, the equilibrium prediction for the Hirshleifer contest differs crucially from that for the standard Tullock contest, as will be discussed further below.

Lemma 2 is obtained from an analysis of the second-order condition at small positive effort levels. As the proof shows, the conclusion is driven by the fact that marginal returns for $a$ contestant in the $n$-player Hirshleifer contest are strictly increasing below the inflection point where the probability of winning the contest equals one half. Thus, while we focus on a particular functional form in this exploratory study, this crucial observation is likely to hold much more generally.

\footnotetext{
${ }^{7}$ Thus, contestant $i$ is active (always active, inactive) if and only if $\mu_{i}^{*}\left(\left(0, V_{i}\right]\right)>0(=1,=0)$.
} 


\section{High and intermediate levels of noise (leading to pure-strategy equilibria)}

This section deals with the case where the decisiveness parameter $\alpha$ is relatively small, which amounts to a substantial degree of noise in the contest technology and tends to result in purestrategy equilibria. Incentives may be so weak that no contestant bothers to exert positive effort (Subsection 3.1). Alternatively, with a bit less noise, one contestant may choose to dominate all other contestants (Subsection 3.2). The findings are discussed and compared to the case of the Tullock contest (Subsection 3.3). Finally, we offer some anecdotal evidence (Subsection $3.4)$.

\subsection{Multilateral peace}

We will say that a PSNE $x^{*}$ reflects multilateral peace if $x_{1}^{*}=\ldots=x_{n}^{*}=0$. By Lemma 2, multilateral peace is the only symmetric PSNE feasible. To understand the conditions for this to be an equilibrium, suppose that all opponents $j \neq i$ of contestant $i$ choose an expense level of zero. Then, contestant $i$ 's expected payoff is given by

$$
\Pi_{i}^{0}\left(x_{i} ; \alpha\right) \equiv \Pi_{i}\left(x_{i}, \mathbf{0}_{n-1}\right)=\frac{V_{i}}{1+(n-1) \exp \left(-\alpha x_{i}\right)}-x_{i}
$$

where $\mathbf{0}_{n-1}=(0, \ldots, 0) \in \mathbb{R}^{n-1}$. Figure 1 outlines the shape of this function. ${ }^{8}$ The illustration suggests that multilateral peace is an equilibrium if and only if there is enough noise, i.e., if and only if $\alpha$ is sufficiently small. This is indeed the case.

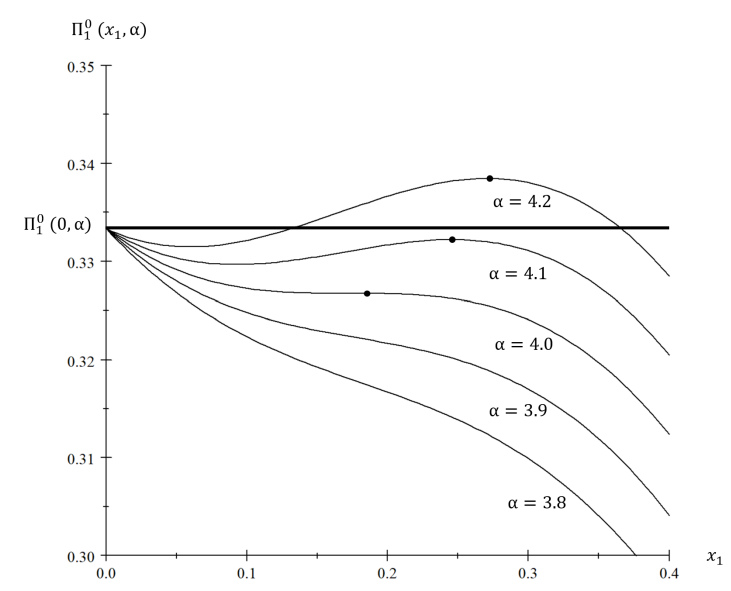

Figure 1. Expected payoff against inactive opponents.

\footnotetext{
${ }^{8}$ Shown is $\Pi_{1}^{0}\left(x_{1} ; \alpha\right)$ as a function of $x_{1}$, for $n=3, V_{1}=1$, and selected values of $\alpha$.
} 
A straightforward examination of marginal payoffs shows that $\Pi_{i}^{0}(\cdot ; \alpha)$ is strictly declining for $\alpha \leq 4 / V_{i}{ }^{9}$ For $\alpha>4 / V_{i}$, however, there is a unique interior local maximum $\widetilde{x}_{i}(\alpha)>0$. In that case, an application of the envelope theorem shows that the payoff at the interior local maximum, $\Pi_{i}^{0}\left(\widetilde{x}_{i}(\alpha), \alpha\right)$, is strictly increasing in $\alpha$. Further, as suggested by Figure 1, for $\alpha>4 / V_{i}$ sufficiently small, remaining inactive is weakly more profitable for contestant $i$ than bidding $\widetilde{x}_{i}(\alpha)$. Arguing along these lines, we arrive at the following result.

Proposition 1. (Multilateral peace) Consider an n-player Hirshleifer contest with valuations $V_{1} \geq \ldots \geq V_{n}>0$. Then, there is a threshold value $\alpha^{*} \equiv \alpha^{*}\left(n, V_{1}\right)>0$ such that:

(i) multilateral peace (i.e., $x_{1}^{*}=\ldots=x_{n}^{*}=0$ ) is an equilibrium if and only if $\alpha \leq \alpha^{*}$;

(ii) in this case, contestant $i$ 's equilibrium payoff equals $\Pi_{i}^{*}=V_{i} / n$, for any $i \in N$;

(iii) the equilibrium is unique (in the set of PSNE) if and only if $\alpha<\alpha^{*} .^{10}$

Proof. See the Appendix.

Thus, for any given number of contestants and any given highest valuation, there is a critical value for the decisiveness parameter $\alpha$ such that multilateral peace is an equilibrium if and only if $\alpha$ remains weakly below that value.

The proof of Proposition 1 reveals that contestant 1's optimality condition is pivotal for the feasibility of multilateral peace, which is intuitively plausible. In particular, the threshold $\alpha^{*}$ may be characterized as the unique solution of the indifference relationship

$$
\Pi_{1}^{0}(0 ; \alpha)=\Pi_{1}^{0}\left(\widetilde{x}_{1}(\alpha) ; \alpha\right)
$$

Solving equation (4) shows that $\alpha^{*}=a(n) / V_{1}$, where $a(n)$ is a constant depending on the number of contestants only. In particular, this shows that the threshold $\alpha^{*}$ is strictly declining $V_{1}$, i.e., multilateral peace becomes less likely as the highest valuation for the contested object rises.

In general, the constant $a(n)$ does not admit a simple analytical expression. However, $a(2)=4$, so that we retrieve Hirshleifer's (1989) classic observation that bilateral peace is a

\footnotetext{
${ }^{9}$ For $\alpha=4 / V_{1}$, there is a saddle point, i.e., marginal payoffs vanish at a positive effort level. Note, however, that $\Pi_{i}^{0}(\cdot ; \alpha)$ is strictly declining also in that case.

${ }^{10}$ We will see below that the equilibrium is also unique within the set of symmetric MSNE for $\alpha \leq \alpha^{*}$.
} 
PSNE if and only if $\alpha \leq 4 / V_{1}$. For $n \geq 3$, the proof of Proposition 1 shows that $a(n) \in\left(4, \frac{n^{2}}{n-1}\right)$. For instance, $a(3) \approx 4.12$. Moreover, $a(n)$ is strictly increasing in $n .^{11}$ Thus, the entry of an additional contestant, unless stronger than contestant 1 , makes it easier to sustain multilateral peace. We will elaborate on this point further later in the paper.

\subsection{One-sided dominance}

Multilateral peace breaks down for $\alpha>\alpha^{*}$. In this case, at least one contestant $i$ (in particular, the strongest contestant $i=1)$ has an incentive to deviate to $x_{i}=\widetilde{x}_{i}(\alpha)>0$. Once contestant $i$ switches to this positive bid level, however, the incentive for any of the other $(n-1)$ contestants to become active are weakened. Thus, a new type of equilibrium candidate arises. We will say that a PSNE $x^{*}$ reflects one-sided dominance by contestant $i$ if $x_{i}^{*}>0$, while $x_{j}^{*}=0$ holds for all other contestants $j \in N \backslash\{i\}$. Our next result characterizes the conditions under which one-sided dominance is an equilibrium.

Proposition 2. (One-sided dominance) Consider an n-player Hirshleifer contest with heterogeneous valuations $V_{1} \geq \ldots \geq V_{n}>0$. Then, there exists $i^{*} \in N$ such that, for any $i \leq i^{*}$, there are threshold values $\alpha_{i}^{*}$ and $\alpha_{i}^{* *}$ such that:

(i) one-sided dominance by contestant $i$ is a PSNE if and only if $\alpha \in\left[\alpha_{i}^{*}, \alpha_{i}^{* *}\right]$;

(ii) in that case, the corresponding efforts are given by $x_{i}^{*}=\widetilde{x}_{i}(\alpha)$ with

$$
\widetilde{x}_{i}(\alpha)=\frac{1}{\alpha} \ln \left(\frac{n-1}{2}\left\{\alpha V_{i}-2+\sqrt{\alpha V_{i}\left(\alpha V_{i}-4\right)}\right\}\right),
$$

and by $x_{j}^{*}=0$ for all $j \in N \backslash\{i\}$, while expected payoffs are given as

$$
\begin{array}{ll}
\Pi_{i}^{*} & =\left(\frac{1}{2}+\sqrt{\frac{1}{4}-\frac{1}{\alpha V_{i}}}\right) V_{i}-x_{i}^{*}, \\
\Pi_{j}^{*} & =\frac{1}{n-1}\left(\frac{1}{2}-\sqrt{\frac{1}{4}-\frac{1}{\alpha V_{i}}}\right) V_{j} \quad(j \in N \backslash\{i\}) ;
\end{array}
$$

(iii) $\alpha_{1}^{*} \leq \ldots \leq \alpha_{i^{*}}^{*}<\alpha_{i^{*}}^{* *} \leq \ldots \leq \alpha_{1}^{* *}$, with $\alpha_{i}^{*}=\left(V_{1} / V_{i}\right) \alpha^{*}$ for $i \leq i^{*}$;

(iv) there does not exist any PSNE except those identified by Proposition 1 and part (i).

Proof. See the Appendix.

\footnotetext{
${ }^{11}$ The proof is available from the authors upon request. The same applies to details on the numerical examples presented further below.
} 
Part (i) says that, under the conditions stated, there is an interval $\left[\alpha_{i}^{*}, \alpha_{i}^{* *}\right]$ of values for the decisiveness parameter $\alpha$ with the property that one-sided dominance by contestant $i$ is an equilibrium and only if $\alpha$ lies in that interval. For $\alpha<\alpha_{i}^{*}$, contestant $i$ would prefer to bid zero. For $\alpha>\alpha_{i}^{* *}$, however, the strongest contestant in the set $N \backslash\{i\}$ would find it profitable to overbid contestant $i$. In the proof, we show that $\alpha_{i}^{* *}$ is well-defined, using an argument analogous to the one used in the previous subsection to identify $\alpha^{*} .^{12}$

Regarding part (ii), we remark that the necessary first-order condition for the unique interior optimum delivers the effort level of the dominant contestant as $x_{i}^{*}=\widetilde{x}_{i}(\alpha)$. One can easily check that $\widetilde{x}_{i}(\alpha)$ is increasing in $V_{i}$, the intuition being that a higher valuation is equivalent to a lower marginal cost of effort. The comparative statics of the equilibrium with respect to the decisiveness parameter $\alpha$ is less immediate. For instance, a numerical exercise for the case of homogenous valuations reveals that, in the relevant range where the equilibrium exists, the equilibrium effort level for the active contestant, $\widetilde{x}_{1}(\alpha)$, is strictly increasing in $\alpha$ for $n \in\{3,4\}$, hump-shaped in $\alpha$ for $n \in\{5,6\}$, and strictly declining $n \geq 7$. Thus, contrary to intuition, if the number of contestants is small, then a sharper sword may induce the dominating contestant to choose an even higher effort level.

As for the equilibrium payoffs, the active contestant $i$ receives a payoff weakly exceeding the "fair share", i.e., $\Pi_{i}^{*} \geq V_{i} / n$. Indeed, contestant $i$ must find it weakly profitable to depart from multilateral peace. Moreover, as the dominant contestant's expenses and raised probability of winning bite into the total cake available for distribution, less than the fair share is left for each of the subdued contestants (all of which win with identical probability). Thus, $\Pi_{j}^{*}<V_{j} / n$ for any $j \neq i$.

Part (iii) shows that the intervals $\left[\alpha_{i}^{*}, \alpha_{i}^{* *}\right]$ over which one-sided dominance by contestant $i$ is an equilibrium are nested for $i \in\left\{1, \ldots, i^{*}\right\}$, with the interval for a contestant with (strictly) higher valuation (strictly) containing the interval for any contestant with a lower valuation.

Finally, part (iv) of the proposition says that there is no PSNE whatsoever for $\alpha>\alpha_{1}^{* *}$. Given that multilateral peace and one-sided dominance are the only feasible types of PSNE by

\footnotetext{
${ }^{12}$ Clearly, each $\alpha_{i}^{* *}=\alpha_{i}^{* *}\left(n, V_{i}, \max _{j \neq i} V_{j}\right)$ is a function of $n, V_{i}$, and $\max _{j \neq i} V_{j}$ only, because the optimality condition of the strongest competitor of contestant $i$ is pivotal for one-sided dominance by $i$ to be an equilibrium.
} 
Lemma 2, this conclusion should be immediate in view of part (i) and Proposition 1.

The comparative statics of equilibrium payoffs is intuitive. Indeed, as seen before, the equilibrium payoff of the active contestant, $\Pi_{i}^{*}=\Pi_{i}^{0}\left(\widetilde{x}_{i}(\alpha) ; \alpha\right)$, is strictly increasing in $\alpha$. On the other hand, the equilibrium payoff of any inactive contestant $j \neq i$ is strictly declining in $\alpha$, as may be easily seen by plugging the explicit solution (5) into $j$ 's payoff function.

A straightforward continuity argument shows that, in general, the identity of the active contestant is undetermined. Thus, the present discussion implies the existence of a multiplicity of payoff-inequivalent PSNE (even in the case of homogeneous valuations).

Corollary 1. (Dominance by a weaker contestant) Let $V_{1} \geq V_{2} \geq \ldots \geq V_{n}>0$, where $n \geq 3$. Suppose that $\alpha \in\left(\alpha_{1}^{*}, \alpha_{1}^{* *}\right)$. Let $i \in\{2, \ldots, n\}$. Then, for $V_{1} / V_{i}$ sufficiently close to one, there exists a PSNE in which contestant $i$ is active, while all the other contestants remain inactive.

Proof. See the Appendix.

\subsection{Discussion}

Taken together, Propositions 1 and 2 offer a fairly comprehensive characterization of the set of PSNE in the Hirshleifer contest. Notably, the striking differences in the equilibrium prediction between the Tullock and Hirshleifer contests extend to the case of $n$ players. In the Tullock contest, inactivity by all players ("multilateral peace") is never an equilibrium because, regardless of the tie-breaking rule in place, at least $(n-1)$ contestants would receive the prize with probability strictly smaller than one. For those contestants, however, a deviation to any small but positive bid would guarantee the prize, in conflict with the equilibrium property. Similarly, just one player being active ("one-sided dominance") is not feasible in a Tullock contest either because the dominating player would always have a strict incentive to lower her positive bid. ${ }^{13}$

\subsection{Historical illustrations}

We conclude this section with some anecdotal evidence. Neither persisting equilibrium of bilateral or multilateral inaction nor its breakdown due to improvement of effectiveness of

\footnotetext{
${ }^{13}$ For an insightful discussion of this argument, which extends to contest success functions that are homogeneous of degree zero, see Corchón (2000, Appendix).
} 
the combat technology could be foreign to military historians. Recall Carl von Clausewitz's classical quote, "defense is the stronger form of fighting than attack. ... I am convinced that the superiority of the defensive is very great, far greater than it appears at first sight. It is this which explains without any inconsistency most periods of inaction that occur in war." (von Clausewitz [1832] 1976, p. 84.) One fundamental reason is what Clausewitz called "friction in war". This insight may not only illuminate Caesar's observation two thousand years ago that his Roman legions and the Gallic opponents repeatedly refused combat under certain circumstances and von Clausewitz's own records of similar episodes at his time. Effectiveness of inaction was also demonstrated in the defense-dominated medieval Europe and World War I (Hirshleifer 2000, p. 786), and even in the modern wars with tanks and airplanes as well (see, e.g., Lawrence 2017, Chapters 2 and 3).

Another illustration is found in ancient China, during the transition of the late Chou (or Zhou) dynasty from the Spring and Autumn Period (770-481 BC) to the Warring States Period (481-221 BC), both with dozens of states coexisting. Largely due to decisive changes in politico-military organization (emergence of counties ("xian") and prefectures ("jun") as units of military recruitment and the concomitant large-scale standing forces) and significant improvements in warfare technologies (in the form of crossbows, cloud ladders, armor and helmets, etc., as well as specially trained cavalry), the relatively peaceful former period was replaced by the far more violent latter (cf. Lewis, 1999). Indeed, military conflicts among the so-called "states organized for warfare" became more frequent, much longer-lasting (easily dragging on for years rather than months), and remarkably deadlier (at times with hundreds of thousands of military casualties). This historical transition, at least one important dimension thereof, might square with our analysis of how an increase in the decisiveness parameter, which measures combat effectiveness in this context, may result in the transformation of a multilateral-inaction Nash equilibrium into an equilibrium with activity. ${ }^{14}$

\footnotetext{
${ }^{14}$ These and other historical examples illustrate also the importance of understanding the nature of coalitions between Hirshleiferian contestants (even if unstable, cf. Footnote 6). While this topic is beyond the scope of the present analysis, we refer the reader to Cubel and Sanchez-Pages (2022), whose analysis captures both between-group contests and within-group distribution.
} 


\section{Low levels of noise (leading to mixed-strategy equilibria)}

For small noise, the pure-strategy equilibria considered in the previous section cease to exist. Intuitively, overbidding an active opponent becomes less risky, so that dominating others becomes very costly. Instead, we find equilibria in which at least one contestant randomizes. We start with the case of homogeneous valuations and examine for it both symmetric equilibria (Subsection 4.1) and asymmetric equilibria (Subsection 4.2). Thereafter, we discuss the case of heterogeneous valuations (Subsection 4.3).

\subsection{Homogeneous valuations: Symmetric equilibria}

We now focus on the important case where valuations are homogeneous, i.e., where $V_{1}=\ldots=$ $V_{n} \equiv V>0$, and equilibria are symmetric. As usual, we call a Nash equilibrium (pure or mixed) symmetric if all players use the same strategy. In this case, it follows from general arguments that each contestant randomizes over the same finite set of bids

$$
y^{(1)}>\ldots>y^{(L)}=0,
$$

where $L \geq 1$, and each $y^{(l)}$ is selected with probability $q^{(l)}>0$, for $l \in\{1, \ldots, L\} .{ }^{15}$ If $L=1$, then the equilibrium is in pure strategies, and necessarily multilateral peace, as seen above. For $L \geq 2$, however, we have a symmetric MSNE.

Below, we offer a general result on the symmetric equilibrium in the $n$-player Hirshleifer contest with homogeneous valuations.

Proposition 3. (Symmetric MSNE) Consider the n-player Hirshleifer contest with homogeneous valuations $V_{1}=\ldots=V_{n} \equiv V>0$. Then the following holds true:

(i) A symmetric equilibrium $\mu^{*}$ exists for any $\alpha>0$;

(ii) $L \geq 2$ if and only if $\alpha>\alpha^{*}$;

(iii) the number $L$ respects the lower bound given by $L \geq\left(\frac{(n-1) \alpha V}{n^{2}}\right)^{\frac{1}{n-1}}$;

(iv) contestant $i$ 's payoff satisfies $\Pi_{i}^{*} \leq \frac{n}{(n-1) \alpha}$.

\footnotetext{
${ }^{15}$ Given the analyticity of the payoff functions on an open neighborhood of the strategy interval, any optimal mixed strategy in the $n$-player Hirshleifer contest has finite support. For a formal statement, see Ewerhart and Sun (2018). Similar techniques have been applied by Ewerhart (2015b, 2021), Sun (2017), and Levine and Mattozzi (2022), in particular.
} 
Proof. See the Appendix.

This proposition characterizes the structure of symmetric MSNE in the $n$-player Hirshleifer contest with homogeneous valuations. Parts (i) and (ii) jointly establish the existence of MSNE with $L \geq 2$ if and only if $\alpha$ strictly exceeds the threshold $\alpha^{*}$ introduced in the previous section. Moreover, multilateral peace (where $L=1$ ) is the unique symmetric MSNE whenever multilateral peace is a PSNE, i.e., for $\alpha \leq \alpha^{*} .{ }^{16}$ Part (iii) puts a general lower bound on the number of mass points in the equilibrium bid distribution. As can be seen, the lower bound on $L$ is strictly increasing and unbounded in $\alpha$. Thus, the number of mass points will ultimately surpass any finite bound as $\alpha \rightarrow \infty$. Remarkably, the payoff bound given in part (iv) does not contain $V$.

The following example illustrates the symmetric MSNE in the simplest case where the support of the symmetric equilibrium strategy has precisely two elements, i.e., $L=2$.

Example 1 (The case $L=2$ ) Let $n \geq 2$. Consider a symmetric equilibrium strategy that places probability $q^{(1)}>0$ on the positive bid $y^{(1)}>0$, and a complementary probability $q^{(2)}=1-q^{(1)}>0$ on the zero bid $y^{(2)}=0$. Then, we have two equations that jointly characterize $y^{(1)}$ and $q^{(1)}$, viz. the first-order condition at the interior bid $y^{(1)}$, and the indifference condition between $y^{(1)}$ and $y^{(2)}$. For instance, if $n=3$, then this type of equilibrium exists for $a \in$ $(4.12,6.98)$, where $\alpha=a / V$. For smaller values of $a$, each contestant would prefer to become inactive. On the other hand, for larger values of $a$, each contestant would wish to deviate to a bid level strictly between zero and $y^{(1)}$.

An interesting conjecture concerns the uniqueness of the symmetric MSNE for $n \geq 3 .{ }^{17}$ The following result may be considered a first step in this direction.

Proposition 4. (Partial uniqueness) Suppose that $\mu_{1}^{*}$ and $\mu_{1}^{* *}$ are two symmetric equilibrium strategies in the $n$-player Hirshleifer contest with homogeneous valuations $V_{1}=\ldots=V_{n} \equiv V>$

\footnotetext{
${ }^{16}$ To prove this, we first show that for $\alpha \leq \alpha^{*}$ and $n \geq 3$, any contestant strictly prefers bidding zero over matching the bid of a single active opponent. The claim then follows by noting that bids are strategic substitutes in the relevant domain.

${ }^{17}$ The case $n=2$ is settled. In fact, there are no asymmetric equilibria in this case either. See Ewerhart and Sun (2018) and Ewerhart (2021).
} 
0. Suppose also that $\mu_{1}^{*}$ and $\mu_{1}^{* *}$ can be ranked in terms of first-order stochastic dominance. Then, $\mu_{1}^{*}=\mu_{1}^{* *}$.

Proof. See the Appendix.

Thus, if multiple symmetric equilibria exist in the Hirshleifer contest with homogeneous valuations, then they are pairwise not comparable in terms of first-order stochastic dominance. The proof of Proposition 4 combines results from the theory of monotone comparative statics with methods from the theory of two-person zero-sum games. It might, therefore, be of some independent interest.

\subsection{Homogeneous valuations: Asymmetric equilibria}

We now turn to the analysis of asymmetric equilibria while keeping the assumption that valuations are homogeneous. As discussed, there are no asymmetric equilibria for $n=2$. For $n \geq 3$, however, the set of asymmetric equilibria even for homogeneous valuations can be quite large, as will be illustrated by three examples.

Example 2. (Two identically randomizing players and one inactive player) Let $n=3$. Suppose that contestants 1 and 2 use an identical mixed strategy that selects $y_{1}^{(1)}=y_{2}^{(1)}>0$ with probability $q_{1}^{(1)}=q_{2}^{(1)} \in(0,1)$ and the zero bid otherwise, while contestant 3 remains inactive. Then, like in Example 1, a first-order condition and an indifference relation jointly characterize $y_{1}^{(1)}$ and $q_{1}^{(1)}$. The resulting MSNE exists for $a \in(4.12,7.01) .{ }^{18}$ For smaller values of $a$, contestants 1 and 2 would wish to reduce expenses to zero. For larger values of $a$, however, both contestants would prefer some bid strictly between $y_{1}^{(1)}$ and zero.

Example 3. (One always active player, one randomizing player, and $(n-2)$ inactive players) Let $n \geq 3$. Suppose that contestant 1 chooses a positive bid $y_{1}^{(1)}>0$ with probability one, while contestant 2 randomizes between the zero bid and overbidding $y_{2}^{(1)}>y_{1}^{(1)}$. ${ }^{19}$ Suppose also that contestants $3, \ldots, n$ all remain inactive. In this case, we have three equilibrium conditions, viz. the respective first-order conditions for $y_{1}^{(1)}$ and $y_{2}^{(1)}$, and the indifference relation

\footnotetext{
${ }^{18}$ E.g., for $a=4.5$, one finds $y_{1}^{(1)}=0.348 \cdot V$ and $q_{1}^{(1)}=0.36$.

${ }^{19} \mathrm{~A}$ minor modification of the argument used in the proof of Lemma 2 shows that it is suboptimal for contestant 2 to bid in the interval $\left(0, y_{1}^{(1)}\right]$.
} 
for contestant 2. For instance, for $n=3$, the MSNE exists for $a \in(4.58,4.66) .{ }^{20}$ For smaller values of $a$, contestant 1 has an incentive to deviate to zero. For larger values of $a$, however, contestant 2 would prefer placing all probability weight on the zero bid.

Example 4. (One always active and two identically randomizing players) Let $n=3$. Suppose that contestant 1 plays a pure strategy $y_{1}^{(1)}>0$, while contestants 2 and 3 identically randomize between the zero bid and $y_{2}^{(1)}=y_{3}^{(1)}>y_{1}^{(1)}$. This MSNE exists for $a \in(4.66,4.86)$. For smaller values of $a$, contestants 2 and 3 would prefer to become inactive, while for larger values of $a$, contestant 1 would wish to withdraw. ${ }^{21}$

\subsection{Heterogenous valuations}

Let $\mu^{*}=\left(\mu_{1}^{*}, \ldots, \mu_{n}^{*}\right)$ be a MSNE in the Hirshleifer contest with valuations $V_{1} \geq \ldots \geq V_{n}>0$. In this case, each contestant $i \in N$ randomizes over a finite set of bids

$$
y_{i}^{(1)}>\ldots>y_{i}^{\left(L_{i}\right)} \geq 0
$$

for some $L_{i} \geq 1$, such that $y_{i}^{\left(l_{i}\right)}$ is selected with probability $q_{i}^{\left(l_{i}\right)}>0$, for $l_{i} \in\left\{1, \ldots, L_{i}\right\}$. Let $L_{i}^{+}$denote the number of positive elements in the support of $\mu_{i}^{*}$. From Lemma 2, we know that $L_{i}^{+}=L_{i}-1$ holds for at least $(n-1)$ players. The following result establishes an upper bound on $L_{i}^{+}$in terms of the cardinalities of the support for the other players.

Proposition 5. (Support inequality) $L_{i}^{+} \leq \prod_{j \neq i} L_{j}$, for any $i \in N$.

Proof. See the Appendix.

This inequality says that the number of pure strategies over which a player can possibly randomize cannot be too heterogeneous across players. The proof exploits the fact that a contestant's first-order condition for the effort levels used with positive probability may be interpreted as a polynomial equation with known degree. The fundamental theorem of algebra then imposes an upper bound on the number of solutions. ${ }^{22}$

\footnotetext{
${ }^{20}$ At $a=4.6$, for instance, we find $y_{1}^{(1)}=0.300 \cdot V, y_{2}^{(1)}=0.513 \cdot V$, and $q_{2}^{(1)}=0.163$.

${ }^{21}$ It may be noted that all our examples of asymmetric MSNE are semi-mixed, i.e., at least one contestant uses a pure strategy. However, in the set-up of Example 4, around $a=4.86$, there is an asymmetric equilibrium in which all three contestants randomize.

${ }^{22}$ This method of proof is restricted to the specific case of the Hirshleifer contest. In Ewerhart (2021), Laplace transforms are used to derive similar inequalities for flexible classes of noise distributions in the case $n=2$.
} 
In the case of equilibria where only one contestant randomizes, while all other contestants use a pure strategy, Proposition 5 implies that the former randomizes between a zero bid and a single positive bid.

Corollary 2. Suppose that $\mu^{*}$ is an equilibrium in the $n$-player Hirshleifer contest with valuations $V_{1} \geq \ldots \geq V_{n}>0$. Suppose that contestant $i \in N$ randomizes strictly while all contestants $j \neq i$ choose a pure strategy. Then, contestant $i$ randomizes between a zero bid and a single positive bid level.

Proof. Immediate from Proposition 5.

Corollary 2 sheds light on the set of equilibria with the property that $L_{j}=1$ for all $j \neq i$. In view of Lemma 2 , at most one contestant $j \neq i$ can be always active. Therefore, there are two cases. In one case, there is indeed one always active player $j$ choosing a pure strategy, as in Example 3 above. In another case, all the players that choose a pure strategy remain inactive, as detailed by the following result.

Proposition 6. (One randomizing player and $(n-1)$ inactive players) Suppose there are $n \geq 3$ contestants with heterogeneous valuations $V_{1} \geq \ldots \geq V_{n}>0$, and let $i \leq i^{*}$. Then, at $\alpha=\alpha_{i}^{*}$, there is a continuum of payoff-inequivalent equilibria in which contestant $i$ randomizes, choosing $y_{i}^{(1)}=\widetilde{x}_{i}\left(\alpha_{i}^{*}\right)>0$ with probability $q_{i}^{(1)} \in[0,1]$, and the zero bid otherwise, while the other $(n-1)$ contestants all remain inactive.

Proof. See the Appendix.

Thus, for $n \geq 3$, a continuum of MSNE exists when the parameter $\alpha$ lies precisely at one of the threshold values $\alpha_{1}^{*}, \ldots, \alpha_{i^{*}}^{*}$. The boundary cases where $q_{1}^{(1)}=0$ and $q_{1}^{(1)}=1$ are special cases of Propositions 1 and 2, respectively. The continuum of equilibria is due to the fact, familiar from the theory of bimatrix games, that the set of beliefs to which a pure strategy is a best response is convex. 


\section{Vanishing levels of noise}

In this section, we study the structure of equilibrium in the limit case of vanishing noise. We start by examining equilibrium bid distributions for $\alpha$ arbitrarily large but still finite.

Proposition 7. (Arbitrarily small noise) Suppose that $V_{1} \geq \ldots \geq V_{n}>0$. Let $\varepsilon>0$ be small. Then, for any $\alpha$ sufficiently large, any equilibrium $\mu^{*}$ of the $n$-player Hirshleifer contest has the following properties:

(i) $y_{i}^{(1)} \in\left(V_{2}-\varepsilon, V_{2}\right)$ for at least two contestants $i \in N$;

(ii) $\Pi_{1}^{*}<V_{1}-V_{2}+\varepsilon$, and $\Pi_{i}^{*}<\varepsilon$ for any $i \in N \backslash\{1\}$;

(iii) either $y_{i}^{(1)}<\varepsilon$ or $y_{i}^{(1)}>V_{2}-\varepsilon$, for any $i \in N$.

(iv) if $V_{1}=V_{2}$, then $\mu_{i}^{*}(\{0\})<\varepsilon$ for at least two contestants $i \in N$.

Proof. See the Appendix.

The proposition provides information about equilibrium strategies and expected payoffs in the case where the noise in the contest technology becomes arbitrarily small.

Part (i) says that at least two contestants bid arbitrarily close to the valuation of the secondranked contestant. ${ }^{23}$ Effort levels approaching $V_{2}$ are intuitively necessary because, without them, every contestant $j$ of valuation $V_{j} \geq V_{2}$ could earn a substantial rent by overbidding the entire population. Moreover, at least two contestants must compete at such levels because a single high bidder would have an incentive to reduce her bids.

Next, part (ii) says that any rent is dissipated except for any single highest-valuation contestant as $\alpha \rightarrow \infty$. Here, the argument is that any bidder earning substantial rents would not bid arbitrarily close to $V_{2}$. But then, as we show, one of the contestants involved in the highest-bid competition would find it strictly beneficial to marginally overbid the said contestant, which is impossible.

Part (iii) shows that, in the limit, everyone either bids up to $V_{2}$ or ultimately becomes inactive. Again, the proof relies on the idea that one of the contestants bidding nearly up to

\footnotetext{
${ }^{23}$ Clearly, when attention is restricted to symmetric MSNE for homogeneous valuations $V_{1}=\ldots=V_{n}=$ $V>0$, then it easily follows that all bidders bid up to $V$.
} 
the valuation of the second-ranked contestant would prefer to slightly overbid any contestant whose highest bid lies in the interval $\left[\varepsilon, V_{2}-\varepsilon\right]$. However, the proof of part (iii) requires an additional argument, as the rent of the lower bidder cannot be assumed to be substantial.

Finally, part (iv) says that, provided that $V_{1}=V_{2}$, the probability of bidding zero vanishes as $\alpha \rightarrow \infty$ for at least two contestants. ${ }^{24}$ Intuitively, too much weight on the zero bid would allow others to profitably overbid.

Thus, overall, the structure of equilibria in the Hirshleifer contest as $\alpha \rightarrow \infty$ is consistent with those of the $n$-bidder all-pay auction. And indeed, the following limit result may be obtained, which extends a result in Levine and Mattozzi (2022) for bilateral contests to any finite number $n \geq 2$ of contestants.

Proposition 8. (Robustness of the APA) Fix valuations $V_{1} \geq \ldots \geq V_{n}>0$. Let $\left\{\alpha_{m}\right\}_{m=0}^{\infty}$ be an increasing and unbounded sequence of parameters in $\mathbb{R}_{>0}$, and let $\left\{\mu^{m}\right\}_{m=0}^{\infty}$ be a sequence of mixed-strategy profiles in $M$, such that $\mu^{m}$ is a MSNE in the n-player Hirshleifer contest with parameter $\alpha_{m}$, for any $m \geq 0$. If $\left\{\mu^{m}\right\}_{m=0}^{\infty}$ converges in distribution to some limit $\mu^{*} \in M$, then $\mu^{*}$ is a MSNE of the all-pay auction.

Proof. See the Appendix.

\section{Large populations}

In this brief section, we change the perspective by keeping the decisiveness parameter of the contest technology fixed while letting the number of contestants grow indefinitely. It turns out that, for any given $\alpha>0$, and valuations bounded by some $\bar{V}>0$, multilateral peace is the unique equilibrium in a Hirshleifer contest with sufficiently many contestants. Intuitively, as $n$ grows indefinitely, it gets harder and harder for a single contestant to sustain a dominating position.

Proposition 9. Let $\alpha>0$ and $\bar{V}>0$ be given. Then, there exists a threshold value $n^{\#}$ such that for any $n>n^{\#}$, multilateral peace is the unique equilibrium in the n-player Hirshleifer contest with parameter $\alpha$ and heterogeneous valuations $\bar{V} \geq V_{1} \geq \ldots \geq V_{n}>0$.

\footnotetext{
${ }^{24}$ Judging on the basis of the all-pay auction, all of these contestants should bid up to $V_{2}$ in the limit, but we have not been able to prove this formally for the difference-form contest.
} 
Proof. See the Appendix.

\section{Conclusion}

In this paper, we have examined the equilibrium set of the $n$-player Hirshleifer contest with heterogeneous valuations, a canonical contest with additive noise. In line with the findings for contests with multiplicative noise such as the Tullock contest, the equilibrium prediction depends heavily on the level of noise in the contest technology. Notwithstanding, the analysis adds strong support to Hirshleifer's (1989) main conclusion, viz. that the nature of the equilibrium prediction is strikingly different across these two cases.

On a more conceptual level, the results obtained in this study appear to be driven by two characteristic features of the Hirshleifer contest technology, as already highlighted in the introduction: (i) Effectiveness of passivity and (ii) increasing returns to marginal effort up to the inflection point of winning with probability one half. When the contest effort is not very effective (namely, the noise of the game is too large), inaction often proves a powerful choice. However, when it considerably matters, the logic that "God is on the side of the larger battalions" is at work, for "(t)here is an enormous gain when your side's forces increase from just a little smaller than the enemy's to just a little larger", as is stressed by Hirshleifer (1989, p. 103). Not surprisingly, when the decisiveness parameter is large, at least two contestants would engage in fierce competition for the prize, intuitively to be on the side favored by nature. However, even in that case, precisely because at least two contestants become very aggressive bidders, the chance available for the others to win becomes rather slim, and therefore the logic of effectiveness of passivity kicks in again. One way to look at the present paper is that it has tried to disentangle these these effects a bit, thereby shedding light on a very plausible (even though less commonly employed) contest format. In any case, we plan to explore the game-theoretic implications of Hirshleifer's assumption further along several dimensions in our future studies. 


\section{Appendix. Proofs}

This Appendix contains technical proofs omitted from the body of the paper. We start by introducing some additional notation.

Additional notation used in the proofs. For bids $x_{i} \geq 0$ and $x_{-i} \in \mathbb{R}_{\geq 0}^{n-1}$, let

$$
p_{i}\left(x_{i}, x_{-i}\right)=\frac{\exp \left(\alpha x_{i}\right)}{\exp \left(\alpha x_{i}\right)+\sum_{j \neq i} \exp \left(\alpha x_{j}\right)}
$$

denote contestant $i$ 's probability of winning. Further, for a bid vector $x \in \mathbb{R}_{\geq 0}^{n}$ entering as an argument of either $\Pi_{i}$ or $p_{i}$, we will alternatively write $x=\left(x_{i}, x_{j}, x_{-i, j}\right)$, where

$$
x_{-i, j}=\left(x_{1}, \ldots, x_{i-1}, x_{i+1}, \ldots, x_{j-1}, x_{j+1}, \ldots, x_{n}\right) \in \mathbb{R}_{\geq 0}^{n-2} .
$$

Proof of Lemma 1. Each player $i$ 's space of pure strategies, $\left[0, V_{i}\right]$, is compact and nonempty. Moreover, payoff functions are continuous. Therefore, the existence of a MSNE follows from Glicksberg's (1952) theorem.

Proof of Lemma 2. To provoke a contradiction, suppose that two contestants $i, j \in N$ with $i \neq j$ are always active in some MSNE $\mu^{*}$. The second derivative of contestant $i$ 's equilibrium payoff with respect to $x_{i}$ is given by

$$
\frac{\partial^{2} \Pi_{i}}{\partial x_{i}^{2}}=\alpha^{2} p_{i}\left(1-p_{i}\right)\left(1-2 p_{i}\right) V_{i}
$$

where we use the shorthand notation $\Pi_{i}=\Pi_{i}\left(x_{i}, x_{-i}\right)$ and $p_{i}=p_{i}\left(x_{i}, x_{-i}\right)$. Clearly, $p_{i} \in(0,1)$ with probability one. In fact $p_{i} \in\left(0, \frac{1}{2}\right)$ with probability one if $i$ plays $x_{i}=0$ against $\mu_{-i}^{*}$. Taking the expectation over $\mu_{-i}^{*}$, and subsequently changing the order of integration and differentiation, one obtains $\partial^{2} \Pi_{i} / \partial x_{i}^{2}>0$ at $x_{i}=0$. Moreover, by continuity, for $\varepsilon>0$ sufficiently small, $\partial^{2} \Pi_{i} / \partial x_{i}^{2}>0$ at any $x_{i} \in(0, \varepsilon)$. Yet by assumption, $\mu_{i}^{*}$ has no mass point at zero. Hence, the lower bound of contestant $i$ 's equilibrium distribution $\mu_{i}^{*}$, denoted by $\underline{y}_{i}$, is positive. A completely analogous argument shows that $\underline{y}_{j}>0$. Therefore, by renaming players if necessary, we may assume without loss of generality that $0<\underline{y}_{i} \leq \underline{y}_{j}$. Consider now the second-order condition at $x_{i}=\underline{y}_{i}$. Clearly, $p_{i}<\frac{1}{2}$ with probability one if either $n \geq 3$ or $\underline{y}_{i}<\underline{y}_{j}$ (or both). Therefore, $n=2$ and $\underline{y}_{1}=\underline{y}_{2}$. Thus, $p_{1} \leq \frac{1}{2}$ at $x_{1}=\underline{y}_{1}$ where the inequality is strict with 
positive probability unless contestant 2 uses a pure strategy. Again, the same argument can be made with the roles of contestants 1 and 2 exchanged. Therefore, $\left(\underline{y}_{1}, \underline{y}_{2}\right)$ is a (symmetric) interior PSNE. But this is impossible, as pointed out by Hirshleifer (1989, p. 107). Hence, at most one contestant is always active.

Proof of Proposition 1. (i) The marginal payoff of contestant $i \in N$ against $x_{-i}=\mathbf{0}_{-i}$ is given as

$$
\frac{\partial \Pi_{i}^{0}\left(x_{i} ; \alpha\right)}{\partial x_{i}}=\frac{\alpha(n-1) X_{i} V_{i}}{\left(X_{i}+n-1\right)^{2}}-1,
$$

where $X_{i}=\exp \left(\alpha x_{i}\right)$. The right-hand side of (12) vanishes at the solutions of the quadratic equation

$$
X_{i}^{2}+\left(2-\alpha V_{i}\right)(n-1) X_{i}+(n-1)^{2}=0
$$

There is no solution for $\alpha<4 / V_{i}$, and precisely one saddlepoint solution $X_{i}=n-1$ for $\alpha=4 / V_{i}$. In the case $\alpha>4 / V_{i}$, there are two solutions, and contestant 1 's payoff function has a unique interior local maximum at

$$
\widetilde{x}_{i}(\alpha)=\frac{1}{\alpha} \ln \left(\frac{n-1}{2}\left\{\alpha V_{i}-2+\sqrt{\alpha V_{i}\left(\alpha V_{i}-4\right)}\right\}\right) .
$$

Moreover, the local maximum of $\Pi_{i}^{0}\left(x_{i} ; \alpha\right)$ at $x_{i}=0$ is globally optimal if and only if $\Pi_{i}^{0}\left(\widetilde{x}_{i}(\alpha) ; \alpha\right) \leq$ $V_{i} / n$. By the envelope theorem,

$$
\begin{aligned}
\frac{\partial \Pi_{i}^{0}\left(\widetilde{x}_{i}(\alpha) ; \alpha\right)}{\partial \alpha} & =\left.\frac{\partial}{\partial \alpha}\left\{\frac{\exp \left(\alpha x_{i}\right) V_{i}}{\exp \left(\alpha x_{i}\right)+n-1}-x_{i}\right\}\right|_{x_{i}=\widetilde{x}_{i}(\alpha)} \\
& =\frac{(n-1) \widetilde{x}_{i}(\alpha) \exp \left(\alpha \widetilde{x}_{i}(\alpha)\right) V_{i}}{\alpha\left(\exp \left(\alpha \widetilde{x}_{i}(\alpha)\right)+n-1\right)^{2}}>0
\end{aligned}
$$

Thus, $\Pi_{i}^{0}\left(\widetilde{x}_{i}(\alpha) ; \alpha\right)$ is strictly increasing in $\alpha$. Next, suppose that $\alpha$ grows above all bounds. Then, contestant $i$ 's marginal payoff at $x_{i}=0$,

$$
\frac{\partial \Pi_{i}^{0}(0 ; \alpha)}{\partial x_{i}}=\frac{\alpha(n-1) V_{i}}{n^{2}}-1
$$

ultimately turns positive, so that eventually $\Pi_{i}^{0}\left(\widetilde{x}_{i}(\alpha) ; \alpha\right)>V_{i} / n$. Thus, for each contestant $i \in N$, there is a unique threshold $\alpha_{i}^{*} \in\left[\frac{4}{V_{i}}, \frac{n^{2}}{(n-1) V_{i}}\right]$ such that bidding $x_{i}=0$ is a best response to $x_{-i}=\mathbf{0}_{-i}$ if and only if $\alpha \in\left(0, \alpha_{i}^{*}\right]$. Thus, multilateral peace is a PSNE if and only if 
$\alpha \in\left(0, \alpha^{*}\right]$, where $\alpha^{*}=\min _{i \in N}\left\{\alpha_{i}^{*}\right\}{ }^{25}$ To see that player 1's optimality condition is pivotal, we show that $\alpha_{1}^{*} \leq \ldots \leq \alpha_{n}^{*}$. Indeed, the valuation ranking $V_{i} \geq V_{j}$ implies

$$
\frac{\Pi_{i}^{0}(\xi ; \alpha)-\Pi_{i}^{0}(0 ; \alpha)}{V_{i}}-\frac{\Pi_{j}^{0}(\xi ; \alpha)-\Pi_{j}^{0}(0 ; \alpha)}{V_{j}}=\left(\frac{1}{V_{j}}-\frac{1}{V_{i}}\right) \xi \geq 0,
$$

for any $\xi \geq 0$. It follows that $\alpha^{*}=\alpha_{1}^{*}$. (ii) The claims regarding $p_{i}^{*}$ and $\Pi_{i}^{*}$ are now immediate. (iii) By Lemma 2, at least $(n-1)$ contestants remain inactive in any PSNE, i.e., there exists $i \in N$ such that $x_{-i}^{*}=\mathbf{0}_{-i}$. However, in slight extension of the argument given in part (i), for $\alpha<\alpha^{*}$, contestant $i$ 's unique best response to $x_{-i}=\mathbf{0}_{-i}$ is $x_{i}=0$. This proves that multilateral peace is unique within the set of PSNE if and only if $\alpha<\alpha^{*}$. The claim follows.

Proof of Proposition 2. (i) Let $\alpha_{i}^{*}$ be defined as in the proof of Proposition 1. We start by showing that one-sided dominance by contestant $i$ is not a PSNE for $\alpha<\alpha_{i}^{*}$. Indeed, for this case, we have seen that $i$ 's unique pure best response to $x_{-i}=\mathbf{0}_{n-1}$ is $x_{i}=0$, which proves the assertion. Next, we claim that one-sided dominance by contestant $i$ is a PSNE for $\alpha \geq \alpha_{i}^{*}$. But if $\alpha \geq \alpha_{i}^{*}$, then $\Pi_{i}^{0}\left(\widetilde{x}_{i}(\alpha) ; \alpha\right) \geq \Pi_{i}^{0}\left(x_{i} ; \alpha\right)$ for any $x_{i} \geq 0$. Thus, contestant $i$ has no incentive to deviate from $x_{i}^{*}=\widetilde{x}_{i}(\alpha)$. To understand the incentives to deviate for any inactive contestant $j \neq i$, consider the payoff function

$$
\widehat{\Pi}_{j}\left(x_{j} ; \alpha\right)=\frac{X_{j} V_{j}}{X_{i}^{*}+X_{j}+n-2}-x_{j},
$$

where $X_{i}^{*}=\exp \left(\alpha x_{i}^{*}\right)$ and $X_{j}=\exp \left(\alpha x_{j}\right)$. As in the proof of Proposition 1, one checks that the contestant with the highest valuation in $N \backslash\{i\}$ is pivotal, therefore, we may assume without loss of generality that $j \in \arg \max _{k \in N \backslash\{i\}} V_{k}$, i.e., that $j=2$ if $i=1$, and $j=1$ if $i \geq 2$. An examination of the derivative $\partial \widehat{\Pi}_{j}\left(x_{j} ; \alpha\right) / \partial x_{j}$ shows that $\widehat{\Pi}_{j}(\cdot ; \alpha)$ is strictly declining for $\alpha \leq 4 / V_{j}$. Moreover, for $\alpha>4 / V_{j}$, solving the necessary first-order condition

$$
\frac{\alpha X_{j}\left(X_{i}^{*}+n-2\right) V_{j}}{\left(X_{i}^{*}+X_{j}+n-2\right)^{2}}=1
$$

for $x_{j}$ shows that there is a unique interior maximum at $x_{j}=\widehat{x}_{j}(\alpha)$, where

$$
\widehat{x}_{j}(\alpha)=\frac{1}{\alpha} \ln \left(\frac{n-2+X_{i}^{*}}{2}\left\{\alpha V_{j}-2+\sqrt{\alpha V_{j}\left(\alpha V_{j}-4\right)}\right\}\right) .
$$

\footnotetext{
${ }^{25}$ For $n \geq 3,(14)$ implies that $\widetilde{x}_{i}(\alpha)>0$ even for $\alpha=4 / V_{i}$, so that $\alpha^{*} \in\left(\frac{4}{V_{1}}, \frac{n^{2}}{(n-1) V_{1}}\right)$ in that case.
} 
Moreover, using the envelope theorem,

$$
\frac{\partial \widehat{\Pi}_{j}\left(\widehat{x}_{j}(\alpha) ; \alpha\right)}{\partial \alpha}=\frac{\widehat{X}_{j}\left(\widehat{x}_{j}(\alpha)(n-2)+\left(\widehat{x}_{j}(\alpha)-\widetilde{x}_{i}(\alpha)\right) X_{i}^{*}\right) V_{j}}{\left(X_{i}^{*}+\widehat{X}_{j}+n-2\right)^{2}}>0,
$$

where $\widehat{X}_{j}=\exp \left(\alpha \widehat{x}_{j}(\alpha)\right)$. On the other hand,

$$
\frac{\partial \widehat{\Pi}_{j}(0 ; \alpha)}{\partial \alpha}=-\frac{\widetilde{x}_{i}(\alpha) X_{i}^{*} V_{j}}{\left(X_{i}^{*}+n-1\right)^{2}}<0 .
$$

Hence, the payoff difference $\widehat{\Pi}_{j}\left(\widehat{x}_{j}(\alpha) ; \alpha\right)-\widehat{\Pi}_{j}(0 ; \alpha)$ is strictly increasing in $\alpha$. Moreover, for obvious economic reasons, $\lim _{\alpha \rightarrow \infty} \widehat{\Pi}_{j}\left(\widehat{x}_{j}(\alpha) ; \alpha\right)=V_{j}-x_{i}^{*}$. Thus, there is a unique threshold value $\alpha_{i}^{* *}$ (note that the index $i$ is indeed correct here because $V_{j}$ is determined through $i$ ) such that

$$
\widehat{\Pi}_{j}\left(\widehat{x}_{j}\left(\alpha_{i}^{* *}\right) ; \alpha_{i}^{* *}\right)=\widehat{\Pi}_{j}\left(0 ; \alpha_{i}^{* *}\right)
$$

There are now two cases. If $\alpha_{i}^{*} \leq \alpha_{i}^{* *}$, then one-sided dominance by contestant $i$ is a PSNE if and only if $\alpha \in\left[\alpha_{i}^{*}, \alpha_{i}^{* *}\right]$. If, however, $\alpha_{i}^{*}>\alpha_{i}^{* *}$, then one-sided dominance by contestant $i$ is not a PSNE. (ii) Equation (5) is derived from the FOC for the dominating contestant, noting that the second solution of the quadratic equation is necessarily a local minimum. The remaining equations are then immediate. (iii) We have seen in the proof of Proposition 1 that $\alpha^{*}=\alpha_{1}^{*} \leq$ $\ldots \leq \alpha_{n}^{*}$. In fact, it is easy to check via suitable substitutions that $\alpha_{1}^{*} V_{1}=\ldots=\alpha_{n}^{*} V_{n}$. Next, we show that $\alpha_{n}^{* *} \leq \ldots \leq \alpha_{1}^{* *}$. For this, let

$$
B_{k}=\frac{1}{2}\left(\alpha V_{k}-2+\sqrt{\alpha V_{k}\left(\alpha V_{k}-4\right)}\right)
$$

for $k \in N$ and $\alpha>4 / V_{k}$. It can be shown that relationship (24) amounts to

$$
J\left(B_{j}, B_{i}\right)=B_{j}+1-\frac{\left(B_{j}+1\right)^{2}}{n B_{j}}-\ln B_{j}-\ln \left(n-2+(n-1) B_{i}\right)=0
$$

holding at $\alpha=\alpha_{i}^{* *}$, where $j$ is determined by $i$, as above. It is easy to check that $J\left(B_{j}, B_{i}\right)$ increases in $B_{j}$ and decreases in $B_{i}$ provided that $B_{i}>1, B_{j}>1$. Therefore, for any $i \geq 3$, we have $J\left(B_{j}, B_{i-1}\right) \geq J\left(B_{j}, B_{i}\right)$ at any relevant $\alpha$ since $V_{i-1} \geq V_{i}$. A similar argument shows that $\alpha_{2}^{* *} \leq \alpha_{1}^{* *}$, which proves the claim. Finally, we show that $\alpha_{1}^{* *} \geq \alpha_{1}^{*}$. For this, given what we already know, it suffices to prove that

$$
\widehat{\Pi}_{2}\left(\widehat{x}_{2}\left(\alpha_{1}^{*}\right) ; \alpha_{1}^{*}\right)-\widehat{\Pi}_{2}\left(0 ; \alpha_{1}^{*}\right) \leq 0 .
$$


So assume for the moment that $\alpha=\alpha_{1}^{*}$. Comparing (14) with (21), one obtains

$$
X_{2} \leq X_{1}^{*} \cdot \frac{n-2+X_{1}^{*}}{n-1}
$$

and hence,

$$
\frac{X_{2}}{X_{1}^{*}+X_{2}+n-2}-\frac{1}{X_{1}^{*}+n-1} \leq \frac{X_{1}^{*}-1}{X_{1}^{*}+n-1} .
$$

Moreover, rewriting in the indifference relationship at $\alpha=\alpha_{1}^{*}$,

$$
\frac{X_{1}^{*} V_{1}}{X_{1}^{*}+n-1}-\widetilde{x}_{1}(\alpha)=\frac{V_{1}}{n}
$$

yields

$$
\frac{\left(X_{1}^{*}-1\right) V_{1}}{X_{1}^{*}+n-1}=\frac{n}{n-1} \widetilde{x}_{1}(\alpha)
$$

Therefore, at $\alpha=\alpha_{1}^{*}$,

$$
\begin{aligned}
\widehat{\Pi}_{2}\left(\widehat{x}_{2}(\alpha) ; \alpha\right)-\widehat{\Pi}_{2}(0 ; \alpha) & =\frac{X_{2} V_{2}}{X_{1}^{*}+X_{2}+n-2}-\frac{V_{2}}{X_{1}^{*}+n-1}-\widehat{x}_{2}(\alpha) \\
& \leq \frac{n}{n-1} \widetilde{x}_{1}(\alpha)-\widehat{x}_{2}(\alpha) \\
& =\frac{1}{\alpha} \ln \left(\frac{n-1}{n-2+X_{1}^{*}}\left(X_{1}^{*}\right)^{\frac{1}{n-1}}\right) .
\end{aligned}
$$

The right-hand side of equation (34) is nonpositive since

$$
\left(X_{1}^{*}\right)^{\frac{1}{n-1}} \leq \frac{n-2+X_{1}^{*}}{n-1},
$$

which is implied by the inequality between the geometric and the arithmetic mean. The claim follows. (iv) This is immediate from Lemma 2 in combination with Proposition 1 and part (i) above.

Proof of Corollary 1. Consider the strategy profile characterized by Proposition 2 where player $i$ is active. It suffices to check that, for $V_{1} / V_{i}$ sufficiently close to unity, contestant 1 has no incentive to overbid contestant $i$, and contestant $i$ has no incentive to become inactive. But this follows from $\alpha_{1}^{*}<\alpha<\alpha_{1}^{* *}$ and the continuity of $\alpha_{i}^{*}$ and $\alpha_{i}^{* *}$ in $V_{1}$ and $V_{i}$.

The following lemma is used in the necessity part of the proof of Proposition 3(ii). Intuitively, the lemma captures the point already mentioned in the body of the paper that, for $\alpha \leq \alpha^{*}(n)$ 
and $n \geq 3$, any Hirshleifer contestant strictly prefers remaining inactive over using the same bid as a single active opponent.

Lemma A.1 Let $n \geq 3, \alpha \leq \alpha^{*}(n, V)$, and $y>0$. Then, $\Pi_{1}\left(0, y, \mathbf{0}_{n-2}\right)>\Pi_{1}\left(y, y, \mathbf{0}_{n-2}\right)$.

Proof. Since $\alpha \leq \alpha^{*}(n)$, we know that $\Pi_{1}\left(0, \mathbf{0}_{n-1}\right) \geq \Pi_{1}\left(y, \mathbf{0}_{n-1}\right)$. Thus,

$$
\frac{V}{n} \geq \frac{Y V}{Y+n-1}-y
$$

where $Y=\exp (\alpha y)$. Hence,

$$
\begin{aligned}
\Pi_{1}\left(0, y, \mathbf{0}_{n-2}\right)-\Pi_{1}\left(y, y, \mathbf{0}_{n-2}\right) & =\frac{V}{Y+n-1}-\frac{Y V}{2 Y+n-2}+y \\
& \geq \frac{V}{Y+n-1}-\frac{Y V}{2 Y+n-2}+\frac{Y V}{Y+n-1}-\frac{V}{n} \\
& =\frac{(n-2)(Y-1)^{2} V}{n(Y+n-1)(2 Y+n-2)}>0 .
\end{aligned}
$$

This proves the claim.

The following lemma establishes an upper bound on the equilibrium payoff for any contestant that uses the zero bid in equilibrium. This lemma will be used in the proofs of Proposition 3(iv) and Lemma A.5.

Lemma A.2 Let $\mu^{*}$ be a MSNE in the $n$-player Hirshleifer contest with valuations $V_{1} \geq \ldots \geq$ $V_{n}>0$. If $i \in N$ satisfies $\mu_{i}^{*}(\{0\})>0$, then $\Pi_{i}^{*} \leq \frac{n}{(n-1) \alpha}$.

Proof. Clearly, $p_{i}\left(0, x_{-i}\right) \leq \frac{1}{n}$ for any $x_{-i} \in \mathbb{R}_{\geq 0}^{n-1}$. Therefore, contestant $i$ 's marginal payoff at $x_{i}=0$ is bounded from below by

$$
\frac{\partial \Pi_{i}}{\partial x_{i}}=\alpha p_{i}\left(1-p_{i}\right) V_{i}-1 \geq \alpha \frac{n-1}{n} p_{i} V_{i}-1=\alpha \frac{n-1}{n} \Pi_{i}-1
$$

Taking expectations with respect to $\mu_{-i}^{*}$, and subsequently exchanging differentiation and integration, one obtains $\partial E_{\mu_{-i}^{*}}\left[\Pi_{i}\right] / \partial x_{i} \geq \frac{(n-1) \alpha}{n} \Pi_{i}^{*}-1$. But $\partial E_{\mu_{-i}^{*}}\left[\Pi_{i}\right] / \partial x_{i} \leq 0$ from the KKT condition at $x_{i}=0$, which proves the claim.

Proof of Proposition 3. (i) The proof is analogous to that of Lemma 1, with Becker and Damianov (2006, Th. 1) replacing Glicksberg's theorem. (ii) (Sufficiency) Suppose that $\alpha>$ 
$\alpha^{*}$. By part (i), there exists a symmetric MSNE. This equilibrium cannot be a PSNE by Proposition 1(i). Hence, $L \geq 2$, as claimed. (Necessity) For $n=2$, the proof follows from the uniqueness result in Ewerhart and Sun (2018). Suppose, therefore, that $n \geq 3$. Take a symmetric MSNE $\mu^{*}$ with support $0=y^{(L)}<y^{(L-1)}<\ldots<y^{(1)}$, where $L \geq 2$. To provoke a contradiction, suppose that $\alpha \leq \alpha^{*}$. Focus on the smallest positive bid, $y^{(L-1)}>0$. By the discussion preceding the statement of Proposition $1, \Pi_{1}\left(y^{(L-1)}, \mathbf{0}_{n-1}\right) \leq \Pi_{1}\left(0, \mathbf{0}_{n-1}\right)$. Next, let $x_{-1}^{+}=\left(y^{(L-1)}, \mathbf{0}_{n-2}\right)$ be such that contestant 2 bids $y^{(L-1)}$ and contestants $3, \ldots, n$ all bid zero. As, for any $i \neq 1$, expenses $x_{1}$ and $x_{i}$ are strategic substitutes with respect to $\Pi_{1}\left(x_{1}, \ldots, x_{n}\right)$ as long as $\exp \left(\alpha x_{1}\right) \leq \sum_{j \neq 1} \exp \alpha x_{j}$, we have

$$
\Pi_{1}\left(y_{L-1}, x_{-1}\right)-\Pi_{1}\left(0, x_{-1}\right) \leq \Pi_{1}\left(y_{L-1}, x_{-1}^{+}\right)-\Pi_{1}\left(0, x_{-1}^{+}\right),
$$

for any profile $x_{-1} \neq \mathbf{0}_{n-1}$ in the support of $\mu_{-1}^{*}$. By Lemma A.1, $\Pi_{1}\left(y_{L-1}, x_{-1}^{+}\right)-\Pi_{1}\left(0, x_{-1}^{+}\right)<$ 0 . Hence, bidding $y^{(L)}=0$ strictly dominates bidding $y^{(L-1)}>0$, a contradiction. (iii) Take a symmetric MSNE $\mu^{*}$ in the $n$-player Hirshleifer contest with parameter $\alpha$. By Lemma 2, there exist bid levels $y^{(1)}>\ldots>y^{(L)} \geq 0$, for some $L \geq 1$, with corresponding probabilities $q^{(1)}, \ldots, q^{(L)} \in[0,1]$, such that each contestant $i \in N$ chooses $y^{(l)}$ with probability $q^{(l)}$, for any $l \in\{1, \ldots, L\}$. Fix $l \in\{1, \ldots, L\}$. By the KKT condition at the optimum $x_{1}=y^{(l)}$, we get for contestant 1 that

$$
\begin{aligned}
1 & \geq \frac{\partial E_{\mu_{-1}^{*}}\left[p_{1}\left(x_{1}, x_{-1}\right) V\right]}{\partial x_{1}} \\
& =\alpha V E_{\mu_{-1}^{*}}\left[p_{1}\left(x_{1}, x_{-1}\right)\left(1-p_{1}\left(x_{1}, x_{-1}\right)\right)\right] \\
& =\alpha V \sum_{l_{2}=1}^{L} \ldots \sum_{l_{n}=1}^{L}\left(\prod_{i=2}^{n} q^{\left(l_{i}\right)}\right) p_{1}\left(x_{1}, y^{\left(l_{2}\right)}, \ldots, y^{\left(l_{n}\right)}\right)\left(1-p_{1}\left(x_{1}, y^{\left(l_{2}\right)}, \ldots, y^{\left(l_{n}\right)}\right)\right) \\
& \geq \alpha V\left(q^{(l)}\right)^{n-1} \frac{1}{n}\left(1-\frac{1}{n}\right) .
\end{aligned}
$$

where the inequality in (45) is obtained by dropping all terms corresponding to scenarios in which at least two contestants use different bid levels. Rewriting yields

$$
q^{(l)} \leq\left(\frac{n^{2}}{\alpha V(n-1)}\right)^{\frac{1}{n-1}}
$$


for any $l \in\{1, \ldots, L\}$. Since $q^{(l)}+\ldots+q^{(L)}=1$, this implies

$$
L \geq\left(\frac{(n-1) \alpha V}{n^{2}}\right)^{\frac{1}{n-1}}
$$

as claimed. (iv) By Lemma 2 and symmetry, we know that $\mu_{i}^{*}(\{0\})>0$ for all $i \in N$. Therefore, the claim follows directly from Lemma A.2.

The following two lemmas are used in the proof of Proposition 4.

Lemma A.3 Let $i, j_{1}, j_{2} \in N$ be such that $i \neq j_{1} \neq j_{2} \neq i$. Then, for any $x_{-\left(j_{1}, j_{2}\right)} \in \mathbb{R}_{\geq 0}^{n-2}$, the mapping $\left(x_{j_{1}}, x_{j_{2}}\right) \mapsto \Pi_{i}\left(x_{1}, \ldots, x_{n}\right)$ exhibits strictly increasing differences in $\left(x_{j_{1}}, x_{j_{2}}\right)$.

Proof. Let $X_{k}=\exp \left(\alpha x_{k}\right)$ and $X=\sum_{k=1}^{n} X_{k}$. Then,

$$
\frac{\partial^{2} \Pi_{i}}{\partial x_{j_{1}} \partial x_{j_{2}}}=\frac{\partial^{2}}{\partial x_{j_{1}} \partial x_{j_{2}}}\left(\frac{X_{i} V_{i}}{X}-x_{i}\right)=\frac{2 \alpha^{2} X_{j_{1}} X_{j_{2}} X_{i} V_{i}}{X^{3}}>0
$$

The claim follows.

Lemma A.4 Suppose that $f\left(x_{1}, \ldots, x_{m}\right)$, with $m \geq 2$, is symmetric and exhibits pairwise weakly increasing differences. Then, for any $y \geq x$,

$$
f(y, \ldots, y) \geq m f(y, x, \ldots, x)-(m-1) f(x, \ldots, x) .
$$

Proof. By induction. For $m=2$, increasing differences imply $f(y, y)-f(y, x) \geq f(x, y)-$ $f(x, x)$, while $f(y, x)=f(x, y)$ by symmetry. Thus, $f(y, y) \geq 2 f(y, x)-f(x, x)$, which is just (49) for $m=2$. Suppose that the claim has been shown for some $m \geq 2$. Take some $f$ with $m+1$ arguments. Then, letting $g\left(x_{1}, \ldots, x_{m}\right)=f\left(y, x_{1}, \ldots, x_{m}\right)$ and $h\left(x_{1}, x_{2}\right)=f\left(x_{1}, x_{2}, x, \ldots, x\right)$, both $g$ and $h$ are symmetric and exhibit pairwise increasing differences. Therefore, using the induction hypothesis (49) for $g$ and $h$, one obtains

$$
\begin{aligned}
g(y, \ldots, y) & \geq m g(y, x, \ldots, x)-(m-1) g(x, \ldots, x) \\
& =m h(y, y)-(m-1) h(y, x) \\
& \geq(m+1) h(y, x)-m h(x, x)
\end{aligned}
$$


Hence, the claim holds for $m+1$, and therefore, for all $m$.

Proof of Proposition 4. Given a mixed strategy profile $\mu=\left(\mu_{1}, \ldots, \mu_{n}\right)$, we denote by

$$
\widetilde{\Pi}_{i}(\mu)=E_{\mu}\left[\Pi_{i}\left(x_{1}, \ldots, x_{n}\right)-\frac{V}{n}+\frac{1}{n-1} \sum_{j \neq i} x_{j}\right]
$$

a normalized version of contestant $i$ 's expected payoff. Clearly,

$$
\sum_{i=1}^{n} \widetilde{\Pi}_{i}(\mu)=0
$$

Let $\boldsymbol{\mu}_{-1}^{*}=(\underbrace{\mu_{1}^{*}, \ldots, \mu_{1}^{*}}_{(n-1) \text { times }})$, and $\widehat{\boldsymbol{\mu}}_{-1}^{*}=(\mu_{1}^{* *}, \underbrace{\mu_{1}^{*}, \ldots, \mu_{1}^{*}}_{(n-2) \text { times }})$. By assumption, $\left(\mu_{1}^{*} ; \boldsymbol{\mu}_{-1}^{*}\right)$ is an equilibrium. Hence,

$$
\widetilde{\Pi}_{1}\left(\mu_{1}^{*} ; \boldsymbol{\mu}_{-1}^{*}\right) \geq \widetilde{\Pi}_{1}\left(\mu_{1}^{* *} ; \boldsymbol{\mu}_{-1}^{*}\right)=-(n-1) \widetilde{\Pi}_{1}\left(\mu_{1}^{*} ; \widehat{\boldsymbol{\mu}}_{-1}^{*}\right),
$$

where the equality follows from (54) and the symmetry of the contest. By Lemma A.3 and Echenique (2003, Lemma 4$), f\left(\mu_{-i}\right) \equiv \widetilde{\Pi}_{i}\left(\mu_{i}^{*}, \mu_{-i}\right)$ exhibits pairwise weakly increasing differences w.r.t. to the $(n-1)$ variables $\left\{\mu_{j}\right\}_{j \neq i}$. Hence, using Lemma A.4,

$$
\widetilde{\Pi}_{1}\left(\mu_{1}^{*} ; \widehat{\boldsymbol{\mu}}_{-1}^{*}\right) \leq \frac{1}{n-1} \widetilde{\Pi}_{1}\left(\mu_{1}^{*} ; \boldsymbol{\mu}_{-1}^{* *}\right)+\frac{n-2}{n-1} \widetilde{\Pi}_{1}\left(\mu_{1}^{*} ; \boldsymbol{\mu}_{-1}^{*}\right),
$$

where $\boldsymbol{\mu}_{-1}^{* *}=(\underbrace{\mu_{1}^{* *}, \ldots, \mu_{1}^{* *}}_{(n-1) \text { times }})$. Plugging (56) in (55) yields $(n-1) \widetilde{\Pi}_{1}\left(\mu_{1}^{*} ; \boldsymbol{\mu}_{-1}^{*}\right) \geq-\widetilde{\Pi}_{1}\left(\mu_{1}^{*} ; \boldsymbol{\mu}_{-1}^{* *}\right)$. Hence,

$$
(n-1) \widetilde{\Pi}_{1}\left(\mu_{1}^{*} ; \boldsymbol{\mu}_{-1}^{*}\right) \geq-\widetilde{\Pi}_{1}\left(\mu_{1}^{*} ; \boldsymbol{\mu}_{-1}^{* *}\right) \geq-\widetilde{\Pi}_{1}\left(\mu_{1}^{* *} ; \boldsymbol{\mu}_{-1}^{* *}\right)=(n-1) \widetilde{\Pi}_{1}\left(\mu_{1}^{* *} ; \boldsymbol{\mu}_{-1}^{* *}\right) .
$$

As this holds analogously for the other players, relationship (54) implies that $\widetilde{\Pi}_{i}\left(\mu_{1}^{*} ; \boldsymbol{\mu}_{-1}^{*}\right)=$ $\widetilde{\Pi}_{i}\left(\mu_{1}^{* *} ; \boldsymbol{\mu}_{-1}^{* *}\right)$, for $i \in N{ }^{26}$ Therefore, all inequalities above are equalities. But then, necessarily $\mu_{1}^{*}=\mu_{1}^{* *}$ (this follows from a straightforward extension of Echenique's (2003) result to the case of strictly increasing differences), as has been claimed.

Proof of Proposition 5. The first-order condition for contestant $i$ 's problem reads

$$
\sum_{x_{-j} \in \operatorname{supp}\left(\mu_{-i}\right)}\left(\frac{\alpha X_{i} X_{-i} V_{i}}{\left(X_{i}+X_{-i}\right)^{2}} \prod_{j \neq i} \mu_{j}\left(\left\{x_{j}\right\}\right)\right)=1
$$

\footnotetext{
${ }^{26}$ Another way to see this equation is to note that the normalized payoffs correspond to a symmetric $n$-person zero-sum game in which symmetric equilibrium payoffs are necessarily zero.
} 
where $X_{i}=\exp \left(\alpha x_{i}\right)$ and $X_{-i}=\sum_{j \neq i} \exp \left(\alpha x_{j}\right)$. Multiplying by $\prod_{x_{-j} \in \operatorname{supp}\left(\mu_{-i}\right)}\left(X_{i}+X_{-i}\right)^{2}$ yields a polynomial equation of degree $D=2 \cdot \prod_{j \neq i} L_{j}$ in the unknown $X_{i}$. By the fundamental theorem of algebra, this equation has at most $D$ solutions. But any two neighboring interior maxima are separated by a local minimum. Hence, $E_{\mu_{-i}}\left[\prod_{i}\left(x_{i} ; x_{-i}\right)\right]$ admits at most $\prod_{j \neq i} L_{j}$ interior maxima.

Proof of Proposition 6. By Proposition 1, the claim is true for $q_{i}^{(1)}=0$. Similarly, by Proposition 2 and $n \geq 3$, the claim is true for $q_{i}^{(1)}=1$. Suppose now that $q_{i}^{(1)} \in(0,1)$. Clearly, contestant $i$ is indifferent between her two best responses $x_{i}=\widetilde{x}_{i}\left(\alpha^{*}\right)$ and $x_{i}=0$. It remains to be shown that no contestant $j \neq i$ has an incentive to deviate. Consider a specific deviation $\widehat{x}_{j}>0$. Clearly, contestant $j$ has no incentive to deviate to $x_{j}=\widehat{x}_{j}$ if $x_{i}=0$ (by Proposition 1 ), and $j$ has likewise no incentive to deviate to $x_{j}=\widehat{x}_{j}$ if $x_{i}=\widetilde{x}_{i}\left(\alpha^{*}\right)$ (by Proposition 2). Therefore, $j$ has no incentive to deviate to $x_{j}=\widehat{x}_{j}$ if contestant $i$ randomizes between $x_{i}=0$ and $x_{i}=\widetilde{x}_{i}\left(\alpha^{*}\right)$. As $\widehat{x}_{j}>0$ was arbitrary, this proves the claim.

The following three Lemmas A.5 through A.7 prepare the proof of Proposition 7. The first lemma is an existence result identifying, for $\alpha$ sufficiently large, a contestant bidding arbitrarily close to the value of the prize.

Lemma A.5 Suppose that $V_{1} \geq V_{2}=\ldots=V_{m}>V_{m+1} \geq \ldots \geq V_{n}>0$ (where $V_{n+1}=0$ if $m=n)$. Let $b \in\left[V_{m+1}, V_{m}\right)$. Then, for $\alpha$ sufficiently large, any MSNE $\mu^{*}$ has the property that there is a contestant $i \leq m$ such that

$$
F_{i}(b) \equiv \mu_{i}^{*}([0, b]) \leq\left(\frac{2+b / V_{2}}{3}\right)^{\frac{1}{n-1}} .
$$

Proof. Clearly, for any $b \in\left[V_{m+1}, V_{m}\right)$, we have $F_{j}(b)=1$ for any $j \geq m+1$ (if $m<n$ ). By Lemma 2 , at least $(m-1)$ contestants $i \in\{1, \ldots, m\}$ each bid zero with positive probability in $\mu^{*}$. Then, for each of them,

$$
\prod_{j \neq i} F_{j}(b)=\prod_{j \neq i, j \leq m} F_{j}(b) \leq \frac{\frac{b}{V_{2}}+2}{3},
$$

since otherwise, by bidding $\frac{b+V_{2}}{2}$, contestant $i$ wins an expected payoff approaching

$$
\frac{\frac{b}{V_{2}}+2}{3} \times V_{2}-\frac{b+V_{2}}{2}=\frac{V_{2}-b}{6}>0,
$$


in conflict with Lemma A.2. Thus, there exists at least one $j \neq i$ such that $j \leq m$ and

$$
F_{j}(b) \leq\left(\frac{\frac{b}{V_{2}}+2}{3}\right)^{\frac{1}{m-1}} \leq\left(\frac{\frac{b}{V_{2}}+2}{3}\right)^{\frac{1}{n-1}}
$$

This proves the lemma.

The next two lemmas, Lemma A.6 and A.7, each capture the intuition that, as the contest becomes increasingly decisive, a contestant's probability of winning does not depend on bids that are substantially lower.

Lemma A.6 Let $\delta>0$ and $\varepsilon>0$. Then, for $\alpha$ large enough,

$$
p_{i}\left(x_{i}+\varepsilon, 0, x_{-i, j}\right)-p_{i}\left(x_{i}+\varepsilon, x_{i}, x_{-i, j}\right) \leq \delta
$$

for any $i \in N, x_{i} \geq 0$, and $x_{-i, j} \in \mathbb{R}_{\geq 0}^{n-2}$.

Proof. Writing $X_{-i, j}=\sum_{k \neq i, j} \exp \left(\alpha x_{k}\right)$, we see that

$$
\begin{aligned}
& p_{i}\left(x_{i}+\varepsilon, 0, x_{-i, j}\right)-p_{i}\left(x_{i}+\varepsilon, x_{i}, x_{-i, j}\right) \\
& \quad=\frac{\exp \left(\alpha\left(x_{i}+\varepsilon\right)\right)}{\exp \left(\alpha\left(x_{i}+\varepsilon\right)\right)+1+X_{-i, j}}-\frac{\exp \left(\alpha\left(x_{i}+\varepsilon\right)\right)}{\exp \left(\alpha\left(x_{i}+\varepsilon\right)\right)+\exp \left(\alpha x_{i}\right)+X_{-i, j}} \\
& \quad=\frac{\exp \left(\alpha\left(x_{i}+\varepsilon\right)\right)\left(\exp \left(\alpha x_{i}\right)-1\right)}{\left(\exp \left(\alpha\left(x_{i}+\varepsilon\right)\right)+1+X_{-i, j}\right)\left(\exp \left(\alpha\left(x_{i}+\varepsilon\right)\right)+\exp \left(\alpha x_{i}\right)+X_{-i, j}\right)} \\
& \quad \leq \frac{\exp \left(\alpha x_{i}\right)-1}{\exp \left(\alpha\left(x_{i}+\varepsilon\right)\right)} \\
& \leq \exp (-\alpha \varepsilon) .
\end{aligned}
$$

This proves the claim.

Lemma A.7 Let $\delta>0$. Then, for $\alpha$ large enough, in any MSNE $\mu^{*}$ of the $n$-player Hirshleifer contest, we have

$$
p_{i}\left(y_{j}^{(1)}+\delta, x_{j}, x_{-i, j}\right)>p_{j}^{N, \alpha}\left(y_{j}^{(1)}, x_{i}, x_{-i, j}\right)-\delta,
$$

for all $x_{j} \in \operatorname{supp}\left\{\mu_{j}^{*}\right\}, x_{i} \in \operatorname{supp}\left\{\mu_{i}^{*}\right\}$, and $x_{-i, j} \in \mathbb{R}_{\geq 0}^{n-2}$.

Proof. There are two cases. If $x_{i}>y_{j}^{(1)}$, then $x_{i}>x_{j}$, so that

$$
p_{i}\left(y_{j}^{(1)}+\delta, x_{j}, x_{-i, j}\right)>p_{j}\left(y_{j}^{(1)}, x_{i}, x_{-i, j}\right)>p_{j}\left(y_{j}^{(1)}, x_{i}, x_{-i, j}\right)-\delta .
$$


If, however, $x_{i} \leq y_{j}^{(1)}$, then by Lemma A.6, for $\alpha$ large enough,

$$
p_{i}\left(y_{j}^{(1)}+\delta, x_{j}, x_{-i, j}\right) \geq p_{j}\left(y_{j}^{(1)}+\delta, 0, x_{-i, j}\right)-\delta>p_{j}\left(y_{j}^{(1)}, x_{i}, x_{-i, j}\right)-\delta .
$$

This proves (68).

Proof of Proposition 7. (i) By Lemma A.5, for any $\alpha$ sufficiently large, and any MSNE $\mu^{*}$ in the $n$-player Hirshleifer contest with parameter $\alpha$, there exists a contestant $i \in N$ such that $V_{i} \geq V_{2}$ and $y_{i}^{(1)} \geq V_{2}-\frac{\varepsilon}{2}$. We claim that, possibly after raising $\alpha$ even further, there always exists another contestant $j \neq i$ such that $y_{j}^{(1)}>V_{2}-\varepsilon$. Suppose not. Then, $y_{j}^{(1)} \leq V_{2}-\varepsilon$ for any $j \in N \backslash\{i\}$. But then, by the optimality condition for contestant $i$ 's highest bid $x_{i}=y_{i}^{(1)}>0$,

$$
\begin{aligned}
0 & =\frac{\partial E_{\mu_{-i}^{*}}\left[\Pi_{i}\right]}{\partial x_{i}} \\
& =\alpha V_{i} E_{\mu_{-i}^{*}}\left[p_{i}\left(1-p_{i}\right)\right]-1 \\
& \leq \alpha V_{i} E_{\mu_{-i}^{*}}\left[1-p_{i}\right]-1 \\
& \leq \frac{\alpha(n-1) V_{i}}{\exp (\alpha \varepsilon / 2)+(n-1)}-1
\end{aligned}
$$

As the right-hand side of inequality (74) turns negative for large $\alpha$, we arrive at the desired contradiction. The claim follows. (ii) The idea of the following argument is adapted from Baye et al. (1990). By part (i), there exist at least two $i \in N$ such that $y_{i}^{(1)} \geq V_{2}-\frac{\varepsilon}{2}$. Clearly, this implies $\Pi_{i}^{*}<V_{i}-V_{2}+\frac{\varepsilon}{2}$, provided that $V_{i} \geq V_{2}$. We claim that, possibly after raising $\alpha$ even further, $\Pi_{j}^{*}<\varepsilon$ for any $j$ that does not behave as such. Suppose not. Then, $\Pi_{j}^{*} \geq \varepsilon$ for some $j$. In particular, $y_{j}^{(1)}<V_{j}-\varepsilon$. Suppose that contestant $i$ such that $V_{i}=V_{2}$ and $y_{i}^{(1)} \geq 1-\frac{\varepsilon}{2}$ overbids $j$ using the bid $y_{j}^{(1)}+\delta$, where $\delta>0$ is small. Then, from Lemma A.7, taking expectations over $\mu^{*}$, we see that

$$
E_{\mu_{-i}^{*}}\left[p_{i}\left(y_{j}^{(1)}+\delta, x_{j}, x_{-i, j}\right)\right] \geq E_{\mu_{-j}^{*}}\left[p_{j}^{N, \alpha}\left(y_{j}^{(1)}, x_{i}, x_{-i, j}\right)\right]-\delta,
$$

resulting in $\frac{\varepsilon}{2}>\Pi_{i}^{*} \geq \Pi_{j}^{*}-2 \delta \geq \varepsilon-2 \delta$. However, for $\delta \leq \varepsilon / 4$, this is impossible. The claim follows. (iii) In the case that $V_{1}=V_{2}$, Lemma A.5 implies that there exists a contestant $i$ such that $V_{i}=V_{2}$ and such that

$$
F_{i}\left(V_{2}-\frac{\varepsilon}{2}\right) \leq\left(\frac{\frac{V_{2}-\frac{\varepsilon}{2}}{V_{2}}+2}{3}\right)^{\frac{1}{n-1}}=\left(1-\frac{\varepsilon}{6 V_{2}}\right)^{\frac{1}{n-1}}
$$


holds for any $\varepsilon>0$ such that $\varepsilon<V_{2}-V_{m+1}$ (where we let $V_{m+1}=0$ if $m=n$, of course). Using Benoulli's inequality, one obtains

$$
F_{i}\left(V_{2}-\frac{\varepsilon}{2}\right)<1-\frac{\varepsilon}{6(n-1) V_{2}}
$$

To provoke a contradiction, suppose that $y_{j}^{(1)} \in\left[\varepsilon, V_{2}-\varepsilon\right]$ for some contestant $j \in N$. Note that $\Pi_{j}\left(y_{j}^{(1)}+\alpha^{-\frac{1}{2}}, \mu_{-j}^{*}\right)+\left(y_{j}^{(1)}+\alpha^{-\frac{1}{2}}\right)$ is no smaller than $\Pi_{j}\left(y_{j}^{(1)}, \mu_{-j}^{*}\right)+y_{j}^{(1)}$ on the one hand and approaching at most $V_{j} \cdot \Pi_{k \neq j} F_{k}\left(y_{j}^{(1)}+2 \alpha^{-\frac{1}{2}}\right)$ on the other. Thus, $V_{j} \cdot \Pi_{k \neq j} F_{k}\left(y_{j}^{(1)}+2 \alpha^{-\frac{1}{2}}\right)$ is approaching at least $\Pi_{j}\left(y_{j}^{(1)}, \mu_{-j}^{*}\right)+y_{j}^{(1)}$ for large $\alpha$. Hence,

$$
\begin{aligned}
{\left[1-\frac{\varepsilon}{6(n-1) V_{2}}\right] \prod_{k \neq i, j} F_{k}\left(y_{j}^{(1)}+2 \alpha^{-\frac{1}{2}}\right) } & >F_{i}\left(V_{2}-\frac{\varepsilon}{2}\right) \prod_{k \neq i, j} F_{k}\left(y_{j}^{(1)}+2 \alpha^{-\frac{1}{2}}\right) \\
& \geq F_{i}\left(y_{j}^{(1)}+2 \alpha^{-\frac{1}{2}}\right) \prod_{k \neq i, j} F_{k}\left(y_{j}^{(1)}+2 \alpha^{-\frac{1}{2}}\right) \\
& \geq y_{j}^{(1)} / V_{j}
\end{aligned}
$$

By bidding $y_{j}^{(1)}+\delta$, for any $\delta \in\left(4 \alpha^{-\frac{1}{2}}, \frac{\varepsilon^{2}}{12(n-1) V_{2}}\right)$, contestant $i$ then receives a payoff

$$
\begin{aligned}
\Pi_{i}\left(y_{j}^{(1)}+\delta, \mu_{-i}^{*}\right) & \geq V_{i}\left(\prod_{k \neq i} F_{k}\left(y_{j}^{(1)}+\frac{\delta}{2}\right)\right)-\left(y_{j}^{(1)}+\delta\right) \\
& \geq V_{i}\left(\prod_{k \neq i, j} F_{k}\left(y_{j}^{(1)}+2 \alpha^{-\frac{1}{2}}\right)\right)-\left(y_{j}^{(1)}+\delta\right) \\
& \geq\left[\frac{1}{1-\frac{\varepsilon}{6(n-1) V_{2}}} \times \frac{V_{i}}{V_{j}}-1\right] y_{j}^{(1)}-\delta \\
& >\left[\left(1+\frac{\varepsilon}{6(n-1) V_{2}}\right) \times \frac{V_{i}}{V_{j}}-1\right] y_{j}^{(1)}-\delta \\
& \geq \frac{\varepsilon^{2}}{6(n-1) V_{2}}-\delta \\
& >\frac{\varepsilon^{2}}{12(n-1) V_{2}} .
\end{aligned}
$$

In the case $V_{1}>V_{2}$, for any $\varepsilon<V_{2}-V_{m+1}$, the value of $\Pi_{1}\left(V_{2}-\frac{\varepsilon}{2}, \mu_{-1}^{*}\right)$ is approaching at least $V_{1} \cdot\left(\Pi_{k=2}^{m} F_{k}\left(V_{2}-\frac{\varepsilon}{2}\right)\right)-\left(V_{2}-\frac{\varepsilon}{2}\right)$. By claim (ii),

$$
V_{1} \cdot\left(\Pi_{k=2}^{m} F_{k}\left(V_{2}-\frac{\varepsilon}{2}\right)\right)-\left(V_{2}-\frac{\varepsilon}{3}\right) \leq \Pi_{1}\left(V_{2}-\frac{\varepsilon}{3}, \mu_{-1}^{*}\right)<V_{1}-V_{2}+\frac{\varepsilon}{6}
$$

for large $\alpha$. Thus, $\Pi_{k=2}^{m} F_{k}\left(V_{2}-\frac{\varepsilon}{2}\right)<1-\frac{\varepsilon}{6 V_{1}}$. Consequently, there exists a contestant $i \in$ $\{2, \ldots, m\}$ such that

$$
F_{i}\left(V_{2}-\frac{\varepsilon}{2}\right) \leq\left(1-\frac{\varepsilon}{6 V_{1}}\right)^{\frac{1}{n-1}}
$$


We can then take essentially the same route as above for the case $V_{1}=V_{2}$ to likewise show that $\Pi_{i}\left(y_{j}^{(1)}+\delta, \mu_{-i}^{*}\right)>\frac{\varepsilon^{2}}{12(n-1) V_{1}}$ for any $\delta \in\left(4 \alpha^{-\frac{1}{2}}, \frac{\varepsilon^{2}}{12(n-1) V_{1}}\right)$, an absurdity. (iv) By contradiction. Suppose that $V_{1}=V_{2}$ but, regardless of $\alpha$, there exists a contestant $i$ such that $\mu_{j}^{*}(\{0\}) \geq \varepsilon$ for all $j \neq i$. Then, the respective contestant $i$ could bid slightly above zero and claim a positive rent bounded away from zero as $\alpha \rightarrow \infty$. This, however, conflicts with part (ii) above. This proves the last claim and, hence, the proposition.

Proof of Proposition 8 (Sketch). For any $m \geq 0$ and $i \in N$, we denote by $F_{i}^{m}$ the cumulative distribution function of contestant $i$ 's mixed strategy $\mu_{i}^{m}$ in the MSNE $\mu^{m}$ of the $n$-player Hirshleifer contest with parameter $\alpha_{m}$. Clearly, each contestant can ensure a positive expected payoff by bidding zero. Suppose first that $V_{1}=V_{2}$. Then, for $\varepsilon$ small enough,

$$
V_{i} \cdot \prod_{j \neq i} F_{j}^{m}\left(x_{i}-\varepsilon\right)-x_{i} \geq 0 \quad\left(x_{i} \in \operatorname{supp}\left\{\mu_{i}^{m}\right\}\right)
$$

As there cannot be any mass points in the interior of $[0,1]$ in the limit, this implies

$$
V_{i} \cdot \prod_{j \neq i} F_{j}^{m}\left(x_{i}\right)-x_{i} \geq \varepsilon \quad\left(x_{i} \in \operatorname{supp}\left\{\mu_{i}^{m}\right\}\right) .
$$

On the other hand, as all rents are dissipated in the limit,

$$
V_{i} \cdot \prod_{j \neq i} F_{j}^{m}\left(x_{i}\right)-x_{i} \leq \varepsilon \quad\left(x_{i} \in[0,1]\right) .
$$

Letting $m$ go to infinity yields

$$
V_{i} \cdot \prod_{j \neq i} F_{j}\left(x_{i}\right)-x_{i}=0 \quad\left(x_{i} \in \operatorname{supp} \mu_{i}^{*}\right)
$$

where $F_{j}$ denotes the cumulative distribution function of contest $j$ 's limit strategy $\mu_{j}^{*}$. If $V_{1}>V_{2}$, then it suffices to replace $V_{i}$ by $V_{2}$ throughout in the above reasoning for contestant $i=1$. Thus, the limit $\mu^{*}$ is indeed a MSNE of the all-pay auction.

Proof of Proposition 9. Assume that $n>n^{\#}(\alpha)=\exp \left(\alpha V_{1}\right)+1$. Take a MSNE $\mu^{*}$ in the $n$-player Hirshleifer contest with parameter $\alpha$, and let $i \in N$ and $x_{i} \in \operatorname{supp}\left\{\mu_{i}^{*}\right\}$. Then, exploiting that $\exp \left(\alpha x_{i}\right) \leq \exp \left(\alpha V_{1}\right)<n-1$, contestant $i$ 's bid $x_{i}$ wins against any $x_{-i} \in$ $\operatorname{supp}\left\{\mu_{-i}^{*}\right\}$ with probability $p_{i}\left(x_{i}, x_{-i}\right) \leq \exp \left(\alpha x_{i}\right) /\left(\exp \left(\alpha x_{i}\right)+n-1\right)<\frac{1}{2}$. Hence, as in the proof of Lemma $2, \partial^{2} E_{\mu_{-i}^{*}}\left[\Pi_{i}\right] / \partial x_{i}^{2}>0$. As this implies $x_{i}=0$, the claim follows. 


\section{References}

Alcalde, J., Dahm, M. (2010), Rent seeking and rent dissipation: A neutrality result, Journal of Public Economics 94, 1-7.

Anderson, S.P., De Palma, A., Thisse, J.-F. (1992), Discrete Choice Theory of Product Differentiation, MIT Press.

Baik, K.H. (1998), Difference-form contest success functions and effort levels in contests, European Journal of Political Economy 14, 685-701.

Baye, M.R., Kovenock, D., De Vries, C.G. (1990), The all-pay auction with complete information, Center for Economic Research Working Paper No. 9051.

—, - - — (1996), The all-pay auction with complete information, Economic Theory 8, 291-305.

Becker, J.G., Damianov, D.S. (2006), On the existence of symmetric mixed strategy equilibria, Economics Letters 90, 84-87.

Che, Y. K., Gale, I. (2000), Difference-form contests and the robustness of all-pay auctions, Games and Economic Behavior 30, 22-43.

Corchón, L.C. (2000), On the allocative effects of rent seeking, Journal of Public Economic Theory 2, 483-491.

—, Serena, M. (2018), "Contest theory." Handbook of Game Theory and Industrial Organization, Volume II, Edward Elgar Publishing.

Corcoran, W.J. (1988), Long-run equilibrium and total expenditures in rent-seeking, pp. 95-101 in: Rowley, C.K., Tullison, R.D., Tullock, G., The Political Economy of Rent-Seeking, Springer, Boston, MA.

Cubel, M., Sanchez-Pages, S. (2016), An axiomatization of difference-form contest success functions, Journal of Economic Behavior \& Organization 131, 92-105.

—, - (2020), Generalized difference-form contests, mimeo, University of Bath.

-,- (2022), Difference-form group contests, Review of Economic Design, published online on Sept. 5, 2022. 
Echenique, F. (2003), Mixed equilibria in games of strategic complementarities, Economic Theory 22, 33-44.

Ewerhart, C. (2015a), Contest Success Functions: The Common-Pool Perspective, University of Zurich, ECON \#195 (June).

- (2015b), Mixed equilibria in Tullock contests, Economic Theory 60, 59-71.

- (2021), A typology of military conflict based on the Hirshleifer contest, unpublished working paper, University of Zurich, https://ssrn.com/abstract=4002559.

—, Sun, G.-Z. (2018), Equilibrium in the symmetric two-player Hirshleifer contest: Uniqueness and characterization, Economics Letters 169, 51-54.

Garfinkel, M.R., Skaperdas, S. (2007), Economics of conflict: An overview, Handbook of Defense Economics 2, 649-709.

Glicksberg, I.L. (1952), A further generalization of the Kakutani fixed point theorem, with application to Nash equilibrium points, Proceedings of the American Mathematical Society 3, 170-174.

Hirshleifer, J. (1989), Conflicts and rent-seeking success functions: Ratio vs difference models of relative success, Public Choice 63, 101-112.

- (1995), Theorizing about conflict, Handbook of Defense Economics 1, 165-189.

- (2000), The macrotechnology of conflict, Journal of Conflict Resolution 44, 773-792.

—, Riley, J.G. (1992), The Analytics of Uncertainty and Information, Cambridge University Press.

Jia, H. (2008), A stochastic derivation of the ratio form of contest success functions, Public Choice 135, 125-130.

- Skaperdas, S. (2012), Technologies of conflict, The Oxford Handbook of the Economics of Peace and Conflict, 449-472.

- , 一, Vaidya, S. (2013), Contest functions: Theoretical foundations and issues in estimation, International Journal of Industrial Organization 31, 211-222. 
Konrad, K.A. (2009), Strategy and Dynamics in Contests, Oxford University Press.

Lawrence, C.A. (2017), War by Numbers: Understanding Conventional Combat, Potomac Books, University of Nebraska Press.

Levine, D.K., Mattozzi, A. (2022), Success in contests, Economic Theory 73, 595-624.

Lewis, M.E. (1999), "Warring states", in: Loewe, M., Shaughnessy, E.L. (eds.), The Cambridge History of Ancient China: From the Origin of Civilization to 221 B.C., Cambridge University Press.

Mueller, D.C. (2003), Public Choice III, Cambridge University Press.

Skaperdas, S. (1996), Contest success functions, Economic Theory 7, 283-290.

Sun, G.-Z. (2017), Characterizing the symmetric mixed-strategy equilibrium in rent-seeking contests: A real analysis approach, unpublished working paper, available at: SSRN 2965369.

Tullock, G. (1980), Efficient rent seeking, pp. 91-112 in: Buchanan, J., Tollison, R., and Tullock, G. (eds), Toward a Theory of Rent Seeking Society, Texas A\&M University Press, College Station.

von Clausewitz, C. (1832, reprinted 1976), On War, Princeton University Press. 